\title{
Detection of isotropic cosmic birefringence and its implications for axion-like particles including dark energy
}

\author{
Tomohiro Fujita, ${ }^{1}$ Kai Murai, ${ }^{1,2}$ Hiromasa Nakatsuka, ${ }^{1}$ and Shinji Tsujikawa ${ }^{3}$ \\ ${ }^{1}$ Institute for Cosmic Ray Research, The University of Tokyo, Kashiwa, 277-8582, Japan \\ ${ }^{2}$ Kavli Institute for the Physics and Mathematics of the Universe (WPI), \\ The University of Tokyo, Kashiwa 277-8583, Japan \\ ${ }^{3}$ Department of Physics, Waseda University, 3-4-1 Okubo, Shinjuku, Tokyo 169-8555, Japan
}

\begin{abstract}
We investigate the possibility that axion-like particles (ALPs) with various potentials account for the isotropic birefringence recently reported by analyzing the Planck 2018 polarization data. For the quadratic and cosine potentials, we obtain lower bounds on the mass, coupling constant to photon $g$, abundance and equation of state of the ALP to produce the observed birefringence. Especially when the ALP is responsible for dark energy, it is possible to probe the tiny deviation of dark energy equation of state from -1 through the cosmic birefringence. We also explore ALPs working as early dark energy (EDE), which alleviates the Hubble tension problem. Since the other parameters are limited by the EDE requirements, we narrow down the ALP-photon coupling to $10^{-19} \mathrm{GeV}^{-1} \lesssim g \lesssim 10^{-16} \mathrm{GeV}^{-1}$ for the decay constant $f=M_{\mathrm{pl}}$. Therefore, the Hubble tension and the isotropic birefringence imply that $g$ is typically the order of $f^{-1}$, which is a non-trivial coincidence.
\end{abstract}

\section{CONTENTS}

I. Introduction

II. Cosmic Birefringence by ALP

III. Simple ALP models

A. Quadratic potential

B. Axion potential

IV. Early dark energy

A. Higher-order periodic potentials

B. Rock ' $n$ ' roll model

C. Common features in two EDE models

\begin{tabular}{ll} 
V. Discussion & 15 \\
\hline
\end{tabular}

\begin{tabular}{lr} 
VI. Conclusion & 16 \\
\hline
\end{tabular}

\begin{tabular}{ll} 
Acknowledgements & 17 \\
\hline
\end{tabular}

$\begin{array}{ll}\text { References } & 17\end{array}$

\section{INTRODUCTION}

The Cosmic Microwave Background (CMB) observation has played a crucial role in the development of the modern precision cosmology. The WMAP and Planck satellites determined the various cosmological parameters with high precision and established the standard cosmology based on the $\Lambda$ Cold Dark Matter $(\Lambda \mathrm{CDM})$ model [14]. Recently, however, the novel analysis on the Planck 2018 polarization data indicated a hint of a new ingredient beyond $\Lambda$ CDM. Ref. [5] reported a measurement of the isotropic cosmic birefringence, which excludes the null hypothesis at $99.2 \%$ confidence level (CL). Cosmic birefringence is the rotation of the photon polarization angle [6, 7], and the observation of isotropic cosmic birefringence may imply the presence of new physics.

One of the possible sources of cosmic birefringence is axion or axion-like particle (ALP) with a weak coupling to photon 8 -13. Axion is a hypothetical pseudo-Nambu-Goldstone boson originally introduced to solve the strong $C P$ problem [14-16, and ALPs have been introduced in many extensions of the standard model of particle physics [17, 18. 
Especially, those particles predicted in string theory have the broad range of mass and the couplings to gauge fields [19, 20].

In the presence of ALPs coupled to photons through a Chern-Simons term, the difference of the ALP field along the light path induces cosmic birefringence 6, 7, 21, 23. While the ALP perturbation at the decoupling of photon induces anisotropic birefringence, the background motion of the same field induces isotropic birefringence. Therefore, we are interested in the ALP field whose background value evolves in time after the decoupling of CMB photons. If the ALP field rapidly oscillates during the photon decoupling epoch, its background value around the last scattering surface (LSS) is averaged over the duration of thickness of the LSS and exponentially suppressed. In this case, what is left over for the contribution to polarization angle is the ALP field value today, and hence the isotropic birefringence is significantly suppressed. Therefore, we expect that the ALP field can induce a non-negligible birefringence if its mass is small enough not to oscillate until the last scattering epoch.

In particular, if the ALP mass $m$ is as small as today's Hubble constant $H_{0}$ and the field slowly rolls down the potential until now, the ALP may comprise all or part of dark energy [24 35]. In this case, the corresponding dark energy scenario is called thawing quintessence [36] in which the field equation of state is initially close to -1 and it starts to deviate from -1 only recently. The joint likelihood analysis of Planck 2018 combined with the data of supernovae type Ia and baryon acoustic oscillations showed that today's field equation of state is constrained to be in the range $w_{\phi}<-0.95(95 \% \mathrm{CL})$ [4] with the quintessence prior $w_{\phi} \geq-1$ (see also Refs. [37- 39]). Due to the observational degeneracy of $w_{\phi}$ around -1 , it is generally difficult to distinguish thawing quintessence from the cosmological constant. In other words, the time variation of the ALP field is hard to be detected by the standard distance measurements alone. On the other hand, the cosmic birefringence provides an independent probe for constraining the background dynamics of the ALP field. Even if $w_{\phi}$ is very close to -1, we will show that the existence of the ALP-photon coupling $g$ can explain the observed rotation angle of cosmic birefringence, while satisfying other experimental bounds of $g$.

In the $\Lambda \mathrm{CDM}$ model, there is an observational tension of the $H_{0}$ value between $\mathrm{CMB}$ [4] and local astrophysical measurements at low redshifts (cosmic distance ladder) [40 45], with the significance of 4.4 $\sigma$ [4]. Among various solutions to alleviate this problem [46-60], the modifications of the cosmological dynamics prior to the CMB decoupling epoch dubbed early dark energy (EDE) 61-71] have been in active study. In these scenarios, the energy density of EDE behaves like a cosmological constant at early times and increases the Hubble expansion rate before the last scattering epoch, and then it dilutes away like or faster than radiation. As a result, the presence of EDE reduces the sound horizon at the last scattering and increases $H_{0}$ inferred from the observed temperature anisotropies in CMB. This unique evolution of EDE can be realized if the bottom of the potential is equal to or higher order than the quartic potential. With such a non-linear potential, the ALP oscillation gets slower as the amplitude decreases. Consequently, the aforementioned suppression of cosmic birefringence due to the ALP oscillation during the last scattering epoch is not as severe as the quadratic potential, which drastically changes the prediction of birefringence compared to the conventional ALPs.

In this paper, we investigate the possibility that the ALPs account for the recently reported isotropic cosmic birefringence with various potentials. First, we examine the case where the ALP comprises a part of the energy component of our universe including dark energy and dark matter by extending the previous work [72] which some of the authors conducted when only the upper bound on the isotropic cosmic birefringence angle was available. We adopt the quadratic potential and cosine potential and discuss the relations among the ALP mass, the ALP energy fraction, initial conditions, and the inferred value of the coupling $g$. In addition, the equation of state is related to the inferred value of $g$. From these relations, we put lower bounds on $g$, the ALP mass, the current ALP abundance, and the equation of state.

We also investigate the isotropic birefringence produced in the proposed EDE scenarios for the first time, while the earlier work studied the anisotropic birefringence in an EDE model [73. We study two typical models of EDE with higher-order periodic potentials and power-law potentials. Since the ALP mass and initial conditions are restricted by the requirement that the ALP works as EDE, we can infer the ALP parameters with less free parameters than the former case. In particular, for higher-order periodic potentials, the inferred $g$ value and the decay constant $f$ satisfy the non-trivial relation $g f=\mathcal{O}(1)$ for $f=M_{\mathrm{pl}}$.

While we mainly focus on the background ALP field inducing the isotropic birefringence in this paper, the ALP fluctuation at the observer also contributes to it. We also discuss the latter effect.

This paper is organized as follows. In Sec. II] we briefly review the cosmic birefringence induced by the ALPs. On using the recent observed angle of cosmic birefringence, we identify the inferred ALP parameters for the simple ALP models in Sec. III and for the EDE models in Sec. [IV] We discuss other possible contributions to the isotropic birefringence in Sec. V] We conclude in Sec. VI 


\section{COSMIC BIREFRINGENCE BY ALP}

In this paper, we consider an ALP field $\phi$ coupled to photons with the electromagnetic tensor $F_{\mu \nu}$. The gravitational sector is described by general relativity with the Ricci scalar $R$. The action in such theories is given by

$$
\mathcal{S}=\int \mathrm{d}^{4} x \sqrt{-\tilde{g}}\left[\frac{M_{\mathrm{pl}}^{2}}{2} R-\frac{1}{2} g^{\mu \nu} \partial_{\mu} \phi \partial_{\mu} \phi-V(\phi)-\frac{1}{4} F_{\mu \nu} F^{\mu \nu}+\frac{1}{4} g \phi F_{\mu \nu} \tilde{F}^{\mu \nu}\right],
$$

where $\tilde{g}$ is the determinant of metric tensor $g_{\mu \nu}, M_{\mathrm{pl}}=2.435 \times 10^{18} \mathrm{GeV}$ is the reduced Planck mass, $V(\phi)$ is the ALP potential, $g$ is the ALP-photon coupling constant, and $\tilde{F}^{\mu \nu}$ is the dual of electromagnetic tensor. It is known that a linearly polarized photon propagating under the influence of ALP field rotates its polarization plane, because of the parity-violating nature of the ALP and its coupling to photon [6, 7, 21, 23. To observe this phenomenon, the CMB photon is an ideal target. When we observe CMB photons emitted at the last scattering surface (LSS), the rotation angle $\alpha$ of their polarization plane depends on the difference of ALP field values between the observer ("obs") and the LSS. Since this rotation is caused by the ALP-photon coupling, $\alpha$ is also proportional to the coupling constant $g$. The rotation angle $\alpha$ of the CMB photon coming from a direction on the sky (denoted as a unit vector $\hat{\boldsymbol{n}}$ ) is given by 74

$$
\alpha(\hat{\boldsymbol{n}})=\frac{g}{2}\left[\phi\left(t_{0}, \mathbf{0}\right)-\phi\left(t_{\mathrm{LSS}}, d_{\mathrm{LSS}} \hat{\boldsymbol{n}}\right)\right]
$$

where $\mathbf{0}$ is the position of the observer, $t_{0}$ is the present time, $t_{\mathrm{LSS}}$ is the last scattering time, and $d_{\mathrm{LSS}}$ is a distance to the LSS. In the following, we use the subscripts "0" and "LSS" as the values today and at the LSS, respectively. As $\phi$ depends on both time and space, we decompose it into the background and perturbed parts, as,

$$
\begin{aligned}
\phi\left(t_{\mathrm{LSS}}, d_{\mathrm{LSS}} \hat{\boldsymbol{n}}\right) & =\bar{\phi}_{\mathrm{LSS}}+\delta \phi_{\mathrm{LSS}}, \\
\phi\left(t_{0}, \mathbf{0}\right) & =\bar{\phi}_{\mathrm{obs}}+\delta \phi_{\mathrm{obs}}
\end{aligned}
$$

where a bar represents background values. The rotation angle can be also separated into the isotropic and anisotropic terms, as

$$
\alpha(\hat{\boldsymbol{n}})=\bar{\alpha}+\delta \alpha(\hat{\boldsymbol{n}})=\frac{g}{2}\left(\bar{\phi}_{\mathrm{obs}}-\bar{\phi}_{\mathrm{LSS}}+\delta \phi_{\mathrm{obs}}\right)-\frac{g}{2} \delta \phi_{\mathrm{LSS}}(\hat{\boldsymbol{n}})
$$

Recently, re-analyzing the observational data of Planck satellite, Ref. [5] reported the detection of the isotropic birefringence, as

$$
\bar{\alpha}=0.35 \pm 0.14 \mathrm{deg}
$$

Since the null result is excluded at $99.2 \%$ CL, it provides a fascinating hint of new phenomena beyond the standard $\Lambda$ CDM cosmology. On the other hand, the anisotropic birefringence has not yet been detected and only some constraints were derived [75, 76]. Inspired by these latest observations on $\alpha$, we focus on the isotropic birefringence and consider models in which the background ALP field explains the observed value of $\bar{\alpha}$ through the background dynamics, $\bar{\alpha}=\frac{g}{2}\left(\bar{\phi}_{\mathrm{obs}}-\bar{\phi}_{\mathrm{LSS}}\right)$. It should be noted that $\delta \phi_{\mathrm{obs}}$ also contributes to $\bar{\alpha}$ and may be able to account for the observed value by itself. However, since the ALP fluctuations, $\delta \phi_{\text {LSS }}$ and $\delta \phi_{\text {obs }}$, have the same origin and are tightly connected, one should be careful not to violate the observational constraint on $\delta \alpha$, when seeking the possibility of $\bar{\alpha} \simeq g \delta \phi_{\text {obs }} / 2$. In fact, we will see that its contribution is constrained as $|\bar{\alpha}| \leq 0.13$ deg in the simplest case. We will further discuss this issue in Sec. V.

In order to extract constraints on $g$ from the observed value of $\bar{\alpha}$ in Eq. (6), we need to solve the time evolution of $\bar{\phi}(t)$ together with the Friedmann equation. We study the background cosmological dynamics on the spatially flat Friedmann-Lemaître-Robertson-Walker space-time with the line element,

$$
\mathrm{d} s^{2}=-\mathrm{d} t^{2}+a^{2}(t) \delta_{i j} \mathrm{~d} x^{i} \mathrm{~d} x^{j}
$$

where $a(t)$ is the time-dependent scale factor. Then, the scalar field obeys

$$
\ddot{\bar{\phi}}+3 H \dot{\bar{\phi}}+V_{, \bar{\phi}}(\bar{\phi})=0
$$

where $V_{\bar{\phi}}=\mathrm{d} V / \mathrm{d} \bar{\phi}$, a dot represents the derivative with respect to $t$, and $H \equiv \dot{a} / a$ is the Hubble expansion rate. The energy density and pressure of the ALP field are given, respectively, by $\rho_{\phi}=\dot{\phi}^{2} / 2+V(\phi)$ and $P_{\phi}=\dot{\phi}^{2} / 2-V(\phi)$. The associated density parameter and equation of state are

$$
\tilde{\Omega}_{\phi}=\frac{\rho_{\phi}}{3 M_{\mathrm{pl}}^{2} H^{2}}, \quad \tilde{w}_{\phi}=\frac{P_{\phi}}{\rho_{\phi}}
$$


where we denote their today's values as $\Omega_{\phi}$ and $w_{\phi}$, respectively.

To solve Eq. (8) for $\bar{\phi}$, we need to know the evolution of $H$ from the late radiation era to today. For this purpose, we take the energy densities of radiation, nonrelativistic matter, and cosmological constant into account, which are denoted as $\rho_{r}, \rho_{M}$, and $\rho_{\Lambda}$ respectively. They obey the continuity equations,

$$
\rho_{I}+3 H\left(1+w_{I}\right) \rho_{I}=0, \quad(I=r, M, \Lambda),
$$

where $w_{r}=1 / 3, w_{M}=0$, and $w_{\Lambda}=-1$. The Friedmann equation is given by

$$
3 M_{\mathrm{pl}}^{2} H^{2}=\rho_{r}+\rho_{M}+\rho_{\Lambda} .
$$

When the ALP field is nearly frozen by the Hubble friction, $\tilde{w}_{\phi}$ is close to -1 . After $\bar{\phi}$ starts to oscillate around the minimum of a quadratic potential, it behaves as a dust with the averaged equation of state $\tilde{w}_{\phi} \simeq 0$. Then, the energy density of ALP field can be incorporated into either $\rho_{\Lambda}$ or $\rho_{M}$ in Eq. (11).

To compute an effective field value $\langle\bar{\phi}\rangle_{\text {LSS }}$ at the LSS, we also take into account the effect of finite thickness of the LSS. If the time variation of $\bar{\phi}$ is significant around the decoupling epoch of photons, it is not trivial to identify the value of $\langle\bar{\phi}\rangle_{\text {LSS }}$. Indeed, the ALP field with a mass larger than $H$ at the LSS starts to oscillate before the decoupling epoch. A natural way to define the effective field value for the cosmic birefringence in CMB is to take a time average of $\bar{\phi}(t)$ as $[72,73$.

$$
\langle\bar{\phi}\rangle_{\mathrm{LSS}}=\int \mathrm{d} T \mathcal{V}(T) \bar{\phi}(t(T)) .
$$

In Eq. 12 the ALP field is weighed by a visibility function $\mathcal{V}(T)$. This function describes the probability density that a CMB photon, now observed, is scattered at the temperature $T$. We approximate $\mathcal{V}(T)$ by a Gaussian function,

$$
\mathcal{V}(T) \simeq \frac{1}{\sqrt{2 \pi} \sigma_{T}} \exp \left[-\frac{\left(T-T_{L}\right)^{2}}{2 \sigma_{T}^{2}}\right],
$$

where $T_{L}=2941 \mathrm{~K}$ and $\sigma_{T}=248 \mathrm{~K}$ are the numerical fitting parameters [77. If $\bar{\phi}$ is nearly frozen around the decoupling epoch, then $\langle\bar{\phi}\rangle_{\mathrm{LSS}}$ is almost equivalent to $\bar{\phi}$ at $T=T_{L}$.

If the field exhibits rapid oscillations between positive and negative values around the LSS, however, $\langle\bar{\phi}\rangle_{\text {LSS }}$ practically approaches 0 by taking the time average 12 . This is especially the case for the ALP mass $m$ much larger than the Hubble expansion rate $H$ at the LSS. In such a case, even though the amplitude of $\bar{\phi}$ today is smaller than that at the LSS, $\left|\langle\bar{\phi}\rangle_{\text {LSS }}\right|$ is suppressed relative to $\left|\bar{\phi}_{\text {obs }}\right|$ and hence the dominant contribution to $\bar{\alpha}$ comes from $\bar{\phi}_{\text {obs. }}$. In Sec. III. we will discuss the mass range of ALP field in which $\left|\langle\bar{\phi}\rangle_{\text {LSS }}\right|$ becomes smaller than $\left|\bar{\phi}_{\text {obs }}\right|$.

In summary, we will address the possibility to account for the reported value of isotropic birefringence, Eq. (6), by considering several ALP potentials. The background value of $\alpha$ is computed according to

$$
\bar{\alpha}=\frac{g}{2} \Delta \bar{\phi} \equiv \frac{g}{2}\left(\bar{\phi}_{\mathrm{obs}}-\langle\bar{\phi}\rangle_{\mathrm{LSS}}\right) .
$$

On using Eq. (10), the Friedmann Eq. (11) can be expressed as

$$
H(a)=H_{0} \sqrt{\Omega_{M} a^{-4}\left(a+a_{\mathrm{eq}}\right)+\Omega_{\Lambda}},
$$

where $H_{0}$ is today's Hubble constant, which is given by

$$
H_{0}=2.1331 \times 10^{-33} h \mathrm{eV} .
$$

We take the dimensionless constant $h$ to be 0.677 . In Eq. (15), $\Omega_{M}$ and $\Omega_{\Lambda}$ are today's density parameters of nonrelativistic matter and cosmological constant, respectively. We adopt the values $\Omega_{M}=0.31$ and $\Omega_{\Lambda}=0.69$ in our numerical simulation. We also choose the value $a_{\mathrm{eq}}=1 / 3400$ for the scale factor at radiation-matter equality, where $a=1$ today. The central temperature $T_{L}=2941 \mathrm{~K}$ in Eq. $13 \mathrm{p}$ corresponds to the scale factor $a_{L}=T_{0} / T_{L}=$ $9.266 \times 10^{-4}$, where we used today's temperature $T_{0}=2.725 \mathrm{~K}$. From Eq. 15, the Hubble parameter $H_{\mathrm{LSS}}$ at the peak of Gaussian distribution 13 can be estimated as

$$
H_{\mathrm{LSS}} \simeq 3.3 \times 10^{-29} \mathrm{eV} .
$$


When we study the background dynamics of the ALP field, there is a typical constant mass scale $m$ whose energy scale is related to the second derivative $V_{, \bar{\phi} \bar{\phi}}$. For the numerical purpose, we write the field Eq. 8 in the form,

$$
\bar{\phi}^{\prime \prime}+3 \frac{H_{0}}{m} \sqrt{\Omega_{M} a^{-4}\left(a+a_{\text {eq }}\right)+\Omega_{\Lambda}} \bar{\phi}^{\prime}+\frac{V_{, \bar{\phi}}}{m^{2}}=0,
$$

where a prime represents a derivative with respect to the dimensionless variable $\tau \equiv m t$. Since the temperature has the dependence $T \propto a^{-1}$, the integral 12 can be expressed as $\langle\bar{\phi}\rangle_{\mathrm{LSS}}=\int_{0}^{\tau_{0}} \mathrm{~d} \tau H T \mathcal{V} \bar{\phi} / m$. Then, from the past to today, we need to integrate the following differential equation,

$$
\langle\bar{\phi}\rangle_{\mathrm{LSS}}^{\prime}=\frac{H_{0}}{m} \frac{T_{0}}{a} \sqrt{\Omega_{M} a^{-4}\left(a+a_{\mathrm{eq}}\right)+\Omega_{\Lambda}} \mathcal{V}\left(\frac{T_{0}}{a}\right) \bar{\phi} .
$$

For a given potential $V$ and initial conditions, we will integrate Eqs. (15), (18), and $(19)$ to obtain the values of $\bar{\phi}_{\text {obs }}$ and $\langle\bar{\phi}\rangle_{\text {LSS }}$. We will choose one of the initial conditions of the ALP field to be $\bar{\phi}^{\prime}(\tau=0)=0$.

\section{SIMPLE ALP MODELS}

In this section, we compute how much the isotropic birefringence is generated for two different ALP potentials: (A) $V_{\text {mass }}(\phi)=m^{2} \phi^{2} / 2$ and (B) $V_{\cos }(\phi)=m^{2} f^{2}[1-\cos (\phi / f)]$. We explore the mass region, $10^{-42} \mathrm{eV} \lesssim m \lesssim 10^{-25.5} \mathrm{eV}$, in which the ALP can act as either dark energy or a sub-dominant component of dark matter, depending on its mass. Respecting the constraint on the ALP energy fraction not to ruin the success of the $\Lambda$ CDM cosmology, we will obtain the ALP-photon coupling constant $g$ consistent with the observed isotropic birefringence (6). We also derive lower bounds on the ALP mass and its energy fraction to generate the observed value of $\bar{\alpha}$ in these models.

\section{A. Quadratic potential}

We first consider a quadratic potential given by

$$
V_{\text {mass }}(\phi)=\frac{1}{2} m^{2} \phi^{2} .
$$

In Ref. [72, some of the authors have studied the ALP with the same potential and computed the isotropic and anisotropic birefringence induced by its background and perturbation parts, respectively. In the following, we explain the background calculation in more detail than Ref. [72] and derive the new limits on some parameters by using the observed value of $\bar{\alpha}$.

For the quadratic potential, the ALP field begins to oscillate when $m \simeq H$. If $m \gg H_{\mathrm{LSS}}$, then the ALP field exhibits rapid oscillations around the CMB decoupling epoch. In this case, the LSS value $\langle\bar{\phi}\rangle_{\text {LSS }}$ of Eq. 12 is suppressed to be smaller than $\bar{\phi}_{\text {obs }}$ due to the time averaging of fast oscillations of $\bar{\phi}$. If $m \ll H_{0}$, on the other hand, the Hubble friction does not allow the field to roll down the potential by today, and hence $\Delta \bar{\phi}$ is suppressed. Therefore the cosmic birefringence is most sensitive to the intermediate mass region, $H_{0} \lesssim m \lesssim H_{\text {LSS }}$.

With a given mass $m$, the different choices of initial conditions of the ALP field $\bar{\phi}_{\text {init }}$ not only affects the quantitative estimate of $\Delta \bar{\phi}$ but also today's energy fraction $\Omega_{\phi}$.

For $m \lesssim H_{0}$, the energy density of ALP field can be the source for all of dark energy. As $m$ increases, $\bar{\phi}$ starts to roll down the potential around the redshift $z \lesssim 1$. This leads to the deviation of today's ALP equation of state $w_{\phi}$ from -1 . The likelihood analysis based on the Planck 2018 data [4] with the prior $\tilde{w}_{\phi} \geq-1$ puts the bound $w_{\phi}<-0.95$ at $95 \% \mathrm{CL}$. Applying this constraint to the potential 20 , we find that $\Omega_{\phi}$ can be as large as the density parameter of dark energy $\Omega_{\Lambda}=0.69$ for

$$
m \leq 8.5 \times 10^{-34} \mathrm{eV}
$$

If $m$ is larger than this upper bound, $w_{\phi}$ is larger than -0.95 and hence the energy density of ALP field is not the main source for dark energy.

For $m \geq 10^{-32} \mathrm{eV}$, the ALP field begins to oscillate in the past, and $\tilde{w}_{\phi}$ shows a transition from -1 to 0 by today. In the mass range

$$
10^{-32} \mathrm{eV} \leq m \leq 10^{-25.5} \mathrm{eV}
$$




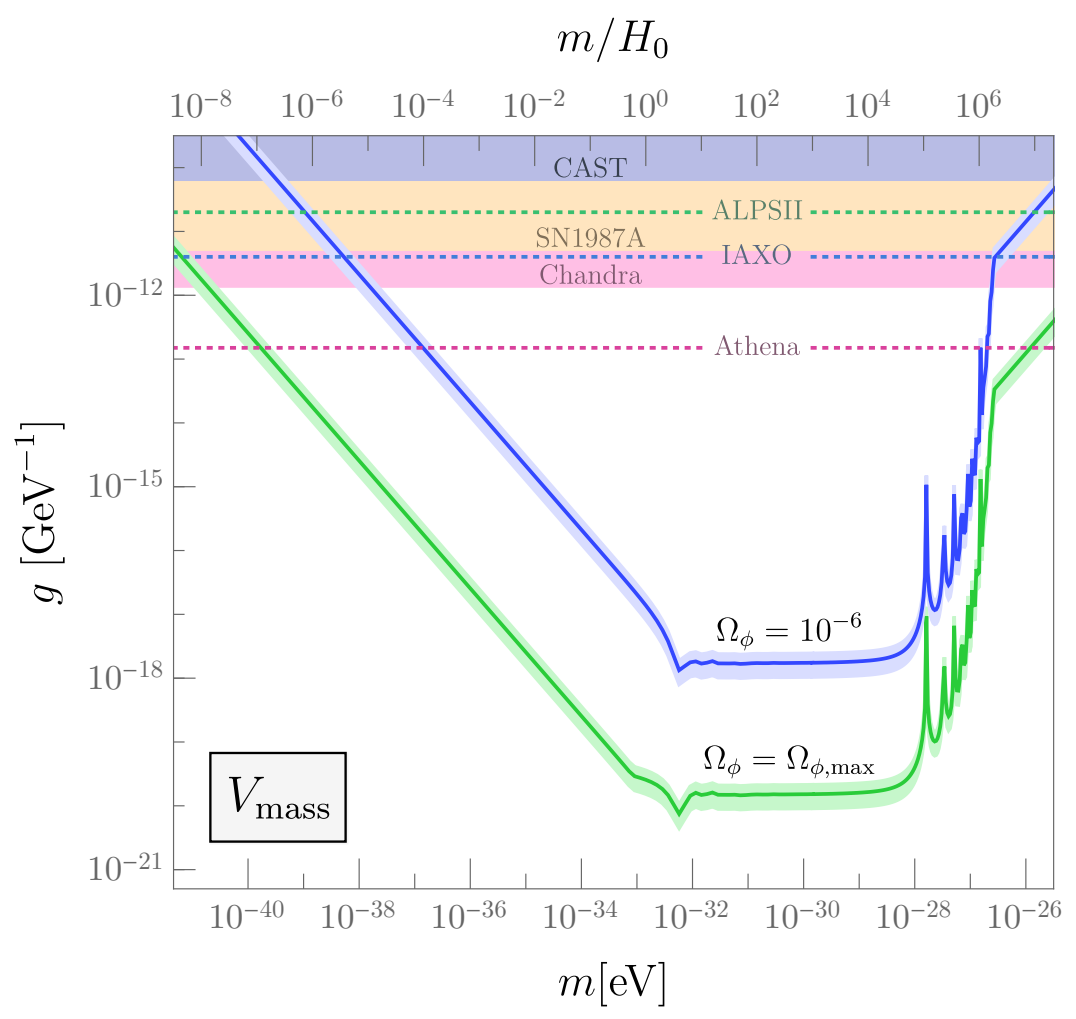

FIG. 1. The ALP-photon coupling constant $g$ inferred by the isotropic birefringence $\bar{\alpha}=0.35 \pm 0.14$ deg versus the ALP mass $m$ for the quadratic potential $V_{\text {mass }}(\phi)=m^{2} \phi^{2} / 2$. The green line corresponds to the maximum energy fraction $\Omega_{\phi}=\Omega_{\phi, \text { max }}$ given in Eq. 23, whereas the blue line shows the case with $\Omega_{\phi}=10^{-6}$. The shaded regions are excluded by the measurements of CAST [79] (blue), SN1987A [80] (orange), and Chandra 81] (pink). We also plot the projected sensitivities of the future experiments, ALPSII [82, IAXO [83, 84, and Athena [85, from top to bottom as dotted lines.

the observations of CMB and large-scale structures put constraints on the ALP with such a transition of $\tilde{w}_{\phi}$, as $\Omega_{\phi} h^{2} \leq 0.006[78$.

From these constraints, we obtain the upper limit of $\Omega_{\phi}$, as

$$
\Omega_{\phi, \max }= \begin{cases}0.69 & \left(m \leq 8.5 \times 10^{-34} \mathrm{eV}\right), \\ 0.006 h^{-2} & \left(10^{-32} \mathrm{eV} \leq m \leq 10^{-25.5} \mathrm{eV}\right) .\end{cases}
$$

In the intermediate mass region $8.5 \times 10^{-34} \mathrm{eV}<m<10^{-32} \mathrm{eV}$, we linearly connect these upper limits of $\Omega_{\phi}$ in the $\log m-\log \Omega_{\phi}$ plane. Note that $\Omega_{\phi, \max }$ increases for the heavier mass region, $m>10^{-25.5} \mathrm{eV}$. The ALP behavior becomes more similar to that of normal dark matter there, and several complications such as the growth of perturbations due to clustering and the shorter oscillation period may not be negligible. To make a conservative argument, we restrict ourselves to the lighter mass region, $m \leq 10^{-25.5} \mathrm{eV}$. In Sec. V], we will further discuss these phenomenological aspects of the higher mass ALP.

With the energy constraint $\Omega_{\phi} \leq \Omega_{\phi, \max }$, we compute the field excursion $\Delta \bar{\phi}$ by numerically solving Eqs. (18) and (19). In Fig. 1, we plot the ALP-photon coupling $g$ which generates the observed isotropic birefringence $\bar{\alpha}=$ $0.35 \pm 0.14 \mathrm{deg}$ for a given $m$. The green line, which corresponds to the maximum ALP energy fraction $\Omega_{\phi}=\Omega_{\phi, \max }$, can be interpreted as the lower bound on $g$, while the blue line corresponds to $\Omega_{\phi}=10^{-6}$. In addition, we also show the current constraints on $g$ with shaded regions and the future sensitivities with dotted lines. CAST [79] and IAXO [83, 84] are axion helioscope experiments, Chandra 81] and Athena 85] are X-ray observatories, and ALPSII [82] is a light shining through a wall style experiment. The ALP-photon coupling is also constrained by the conversion to photons in galactic magnetic fields of axions emitted by SN1987A [80].

As for the behavior of $g$ in Fig. 1, there are three distinct regions depending on the mass $m$. In the following, we will discuss each of them in turn.

For $m \lesssim H_{0}$, the ALP field is nearly frozen until recently, in which case $3 H \dot{\bar{\phi}} \simeq-m^{2} \bar{\phi}$. The field excursion $\Delta \bar{\phi}$ is approximately proportional to $m^{2} \bar{\phi}_{\mathrm{obs}}$, where $\Omega_{\phi} \simeq m^{2} \bar{\phi}_{\mathrm{obs}}^{2} /\left(6 M_{\mathrm{pl}}^{2} H_{0}^{2}\right)$. This means that the rotation angle 14 has 
the dependence,

$$
|\bar{\alpha}| \propto g m^{2}\left|\bar{\phi}_{\text {obs }}\right| \propto g m \sqrt{\Omega_{\phi}}, \quad\left(m \lesssim H_{0}\right),
$$

where the constant of proportionality can be determined numerically.

In the intermediate mass region $H_{0} \lesssim m \lesssim H_{\mathrm{LSS}}, \bar{\phi}$ starts to oscillate at $m \simeq H_{\mathrm{osc}}$, where $H_{\mathrm{osc}}<H_{\mathrm{LSS}}$. The field value at the onset of oscillations (denoted as the scale factor $a_{\text {osc }}$ ) is practically identical to $\langle\bar{\phi}\rangle_{\mathrm{LSS}}$, after which the amplitude of $\bar{\phi}$ decreases as $\Phi \propto a^{-3 / 2}$. In this case $\bar{\phi}_{\text {obs }}$ is negligible relative to $\langle\bar{\phi}\rangle_{\text {LSS }}$, so that $\bar{\alpha} \simeq-g\langle\bar{\phi}\rangle_{\text {LSS }} / 2$. On using the matter-dominated approximation $\left(a \propto H^{-2 / 3}\right)$ to relate $\langle\bar{\phi}\rangle_{\text {LSS }}$ with today's field amplitude $\Phi_{0}$, we obtain

$$
\langle\bar{\phi}\rangle_{\mathrm{LSS}} \simeq\left(\frac{a_{\mathrm{osc}}}{a_{0}}\right)^{-3 / 2} \Phi_{0} \simeq \frac{H_{\mathrm{osc}}}{H_{0}} \Phi_{0} \simeq \frac{m}{H_{0}} \Phi_{0} .
$$

Since $\sqrt{\Omega_{\phi}} \propto m \Phi_{0}$, it follows that

$$
|\bar{\alpha}| \propto g m \Phi_{0} \propto g \sqrt{\Omega_{\phi}}, \quad\left(H_{0} \lesssim m \lesssim H_{\mathrm{LSS}}\right)
$$

which means that $|\bar{\alpha}|$ does not depend on $m$.

For $m \gtrsim H_{\mathrm{LSS}} \simeq 3.3 \times 10^{-29} \mathrm{eV}, \bar{\phi}$ starts to oscillate before the last scattering epoch. Up to the mass range $m \lesssim 2.7 \times 10^{-27} \mathrm{eV} \simeq 80 H_{\mathrm{LSS}},\left|\langle\bar{\phi}\rangle_{\mathrm{LSS}}\right|$ is still larger than $\left|\bar{\phi}_{\mathrm{obs}}\right|$, even though $\langle\bar{\phi}\rangle_{\mathrm{LSS}}$ is suppressed due to the oscillations of $\bar{\phi}$ around the LSS. As $m$ increases, the exponential suppression of $\langle\bar{\phi}\rangle_{\text {LSS }}$ tends to be more significant.

For $m \gtrsim 2.7 \times 10^{-27} \mathrm{eV} \simeq 80 H_{\mathrm{LSS}},\left|\langle\bar{\phi}\rangle_{\mathrm{LSS}}\right|$ becomes smaller than $\left|\bar{\phi}_{\text {obs }}\right|$. In this regime, the rotational angle has the dependence,

$$
|\bar{\alpha}| \propto g\left|\bar{\phi}_{\text {obs }}\right| \propto g m^{-1} \sqrt{\Omega_{\phi}}, \quad\left(m \gtrsim 2.7 \times 10^{-27} \mathrm{eV}\right) .
$$

Numerically, we obtain the constants of proportionality in Eqs. 24], 26), and (27). The resulting approximate expressions in three different regimes are given, respectively, by

$$
g= \begin{cases}1.8 \times 10^{-18} \mathrm{GeV}^{-1}\left(\frac{|\bar{\alpha}|}{0.35 \mathrm{deg}}\right)\left(\frac{\Omega_{\phi}}{\Omega_{\Lambda}}\right)^{-1 / 2}\left(\frac{m / H_{0}}{10^{-2}}\right)^{-1}, & \left(m \lesssim H_{0}\right), \\ 1.5 \times 10^{-20} \mathrm{GeV}^{-1}\left(\frac{|\bar{\alpha}|}{0.35 \mathrm{deg}}\right)\left(\frac{\Omega_{\phi} h^{2}}{0.006}\right)^{-1 / 2}, & \left(H_{0} \lesssim m \lesssim H_{\mathrm{LSS}}\right), \\ 1.8 \times 10^{-12} \mathrm{GeV}^{-1}\left(\frac{|\bar{\alpha}|}{0.35 \mathrm{deg}}\right)\left(\frac{\Omega_{\phi} h^{2}}{0.006}\right)^{-1 / 2}\left(\frac{m / H_{0}}{10^{8}}\right), & \left(m \gtrsim 2.7 \times 10^{-27} \mathrm{eV}\right) .\end{cases}
$$

For the mass range $H_{\mathrm{LSS}}<m \lesssim 2.7 \times 10^{-27} \mathrm{eV}, g$ exponentially increases with $m$, while the dependence of $g$ on $|\bar{\alpha}|$ and $\Omega_{\phi}$ are the same as the third of Eq. 28). As we observe in Fig. 1. the coupling $g$ generating the value $\bar{\alpha}=0.35 \mathrm{deg}$ has the mass dependence $g \propto m^{-1}$ for $m \lesssim H_{0}, g \propto m^{0}$ for $H_{0} \lesssim m \lesssim H_{\mathrm{LSS}}$, and $g \propto m$ for $m \gtrsim 2.7 \times 10^{-27} \mathrm{eV}$.

As $\Omega_{\phi}$ decreases from $\Omega_{\phi \text {,max }}$, the green line in Fig. 1 moves upwards, i.e., to the region of larger values of $g$. Since $g$ is bounded from above by Chandra measurements, the mass region which can explain the observed value of $\bar{\alpha}$ is limited. Combining the first of Eq. 28 with the observational bound by Chandra, $g<1.4 \times 10^{-12} \mathrm{GeV}^{-1}$, we obtain the constraint on the ALP mass, as

$$
m>1.8 \times 10^{-41} \mathrm{eV}\left(\frac{0.69}{\Omega_{\phi}}\right)^{1 / 2}\left(\frac{\bar{\alpha}}{0.35 \mathrm{deg}}\right) .
$$

This result is, to our best knowledge, the first lower mass bound on a dark energy model.

In the literature, the time evolution of $\tilde{w}_{\phi}$ is often used to constrain the (effective) mass of a quintessence field. However, the observational allowed range of $\tilde{w}_{\phi}$ is close to the value -1 as we already mentioned above, so it is difficult to distinguish between quintessence and cosmological constant from the observations of supernovae type Ia and the distant measurements of CMB and baryon acoustic oscillations. In comparison to them, the lower ALP mass bound 29. accomplishes the prominent sensitivity to the time variation of quintessence. In a similar way, one can also derive the upper bound on the ALP mass, which is expected to be around $10^{-25}-10^{-23} \mathrm{eV}$. Since it is beyond the applicable limit of Eq. 23, we leave its detailed calculation for future work.

Furthermore, as we see in Fig. 1 the minimum value of $g$ is taken in the intermediate mass region $H_{0} \lesssim m \lesssim H_{\text {LSS }}$. Applying the Chandra constraint on $g$ to the numerically calculated minimum $g$ for a fixed $\Omega_{\phi}$, we obtain the lower bound on $\Omega_{\phi}$, as

$$
\Omega_{\phi}>9.1 \times 10^{-19}\left(\frac{\bar{\alpha}}{0.35 \mathrm{deg}}\right)^{2}
$$


where the numerical coefficient is a bit different from the one derived by the second line of $(28)$ because the actual minimum $g$ for a fixed $\Omega_{\phi}$ is slightly smaller. It is particularly remarkable that the observation of cosmic birefringence gives rise to an extremely small lower bound of $\Omega_{\phi}$. At the same time, it should be noted that Eq. (30) is derived under the assumption that $\Delta \bar{\phi}$ generates the observed value of $\bar{\alpha}$. The perturbation $\delta \phi_{\text {obs }}$ sourced by an adiabatic mode might provide a significant contribution to $\bar{\alpha}$ through Eq. (5), and then the bound can be subject to change. We will discuss such possibilities in Sec. V.

\section{B. Axion potential}

In this section, we consider the ALP field with a cosine potential,

$$
V_{\cos }(\phi)=m^{2} f^{2}\left[1-\cos \left(\frac{\phi}{f}\right)\right]
$$

where $m$ and $f$ are constants having a dimension of mass. This potential is often used in the context of the QCD axion and ALP [17. Due to the periodicity of the potential, we will consider the case in which the field initial value $\bar{\phi}_{\text {init }}$ is in the range $0 \leq\left|\bar{\phi}_{\text {init }}\right| \leq \pi f$. We also choose $f=M_{\mathrm{pl}}$, but the similar calculation can be performed for arbitrary values of $f$.

The difference from the quadratic potential 200 is that $V_{\cos }(\phi)$ is bounded from above, as $V_{\cos }(\phi) \leq 2 m^{2} f^{2}$. Moreover, the potential (31) has a plateau at $\phi=\pi f$, with the inflection point at $\phi=\pi f / 2$. In the region $\phi \ll f$, the potential approximately reduces to the quadratic one, i.e., $V_{\cos }(\phi) \simeq m^{2} \phi^{2} / 2$. The background ALP field obeys

$$
\ddot{\bar{\phi}}+3 H \dot{\bar{\phi}}+m^{2} f \sin \left(\frac{\bar{\phi}}{f}\right)=0
$$

where $H$ is given by Eq. 15 .

For given values of $\bar{\alpha}$ and $m$, we expect that a larger $\bar{\phi}_{\text {init }}$ leads to a greater $\Delta \bar{\phi}$ or, equivalently, a smaller $g$. However, the choice of a large $\bar{\phi}_{\text {init }}$ can give rise to a large $\Omega_{\phi}$ exceeding the maximum $\Omega_{\phi, \max }$ given in Eq. (23). Due to the non-linear property of the cosine potential, it is difficult to analytically relate $\Omega_{\phi}$ to $\bar{\phi}_{\text {init }}$. Therefore, for each $m$, we numerically scan over $\bar{\phi}_{\text {init }}$ to find minimum values of $g$ satisfying the condition $\Omega_{\phi} \leq \Omega_{\phi, \max }$. The green line in Fig. 2 corresponds to the minimum coupling $g_{\min }$ which accounts for the observed value $\bar{\alpha}=0.35 \pm 0.14 \mathrm{deg}$.

In the left panel of Fig. 3, we also show the theoretical line corresponding to the coupling $g_{\min }$ in the $\left(m, \bar{\phi}_{\text {init }}\right)$ plane as the green line, over the contour of $g$ generating $\bar{\alpha}=0.35 \mathrm{deg}$. The gray region is excluded by the violation of the condition $\Omega_{\phi} \leq \Omega_{\phi, \max }$. For $m \lesssim H_{0}$, the field value that generates $g_{\min }$ is $\bar{\phi}_{g_{\min }} \simeq \pi f / 2$. This is because $\bar{\phi}$ slowly rolls down the potential until now in this mass region and the gradient of the potential is maximum at the inflection point, $\bar{\phi}=\pi f / 2$. For $m \gtrsim H_{0}, \bar{\phi}_{g_{\min }}$ corresponds to $\Omega_{\phi}=\Omega_{\phi \text {, max }}$ because $\bar{\phi}$ begins to oscillate by today, and the larger $\bar{\phi}_{\text {init }}$ is, the greater $\Delta \bar{\phi}$ is.

The observational bound (6) of $\bar{\alpha}$ infers that, for smaller $\bar{\phi}_{\text {init }}$, the coupling $g$ tends to be larger, so that the allowed region is more severely constrained by the Chandra bound. Moreover, in the standard scenario, the homogeneous field $\bar{\phi}$ should be generated by the misalignment mechanism, and $\bar{\phi}_{\text {init }} / f$ is naturally expected to be of order unity. Thus, the initial field displacement in the range $\bar{\phi}_{\text {init }} / f \ll 1$ requires a fine tuning. As a reference, we plot the theoretical line corresponding to the initial condition $\bar{\phi}_{\text {init }} / f=10^{-3}$ as a blue line in Fig. 2 .

The properties of theoretical lines in Fig. 2 look similar to those for the quadratic potential plotted in Fig. 1, but there is the difference in the light mass range $m \lesssim H_{0}$. The third term on the left hand side of Eq. (32) approaches 0 as $\bar{\phi}$ increases toward $\pi f$, so the field excursion $\Delta \bar{\phi}$ does not possess linear dependence in $\bar{\phi}_{\text {init }}$. In comparison to the quadratic potential, the coupling $g$ grows more rapidly with the decrease of $m$. The minimum coupling $g$ has the following relation,

$$
g_{\min }=3.6 \times 10^{-16} \mathrm{GeV}^{-1}\left(\frac{\bar{\alpha}}{0.35 \mathrm{deg}}\right)\left(\frac{m / H_{0}}{10^{-2}}\right)^{-2}, \quad\left(m \lesssim H_{0}\right) .
$$

We note that the axion-photon coupling is typically given by $g=c_{\phi} \alpha_{\mathrm{EM}} / f$, where $\alpha_{\mathrm{EM}} \simeq 1 / 137$ is the fine structure constant and $c_{\phi}=\mathcal{O}(1)$ is a dimensionless constant. Therefore, the tiny coupling constant of order $g \sim 10^{-20} \mathrm{GeV}^{-1}$, which corresponds to $m \sim H_{0}$ in Eq. (33), can be naturally expected for the ALP with $f \sim M_{\mathrm{pl}}$. From Eq. (33), the Chandra experiment gives the mass constraint,

$$
m>1.8 \times 10^{-37} \mathrm{eV}\left(\frac{\bar{\alpha}}{0.35 \mathrm{deg}}\right)^{1 / 2},
$$




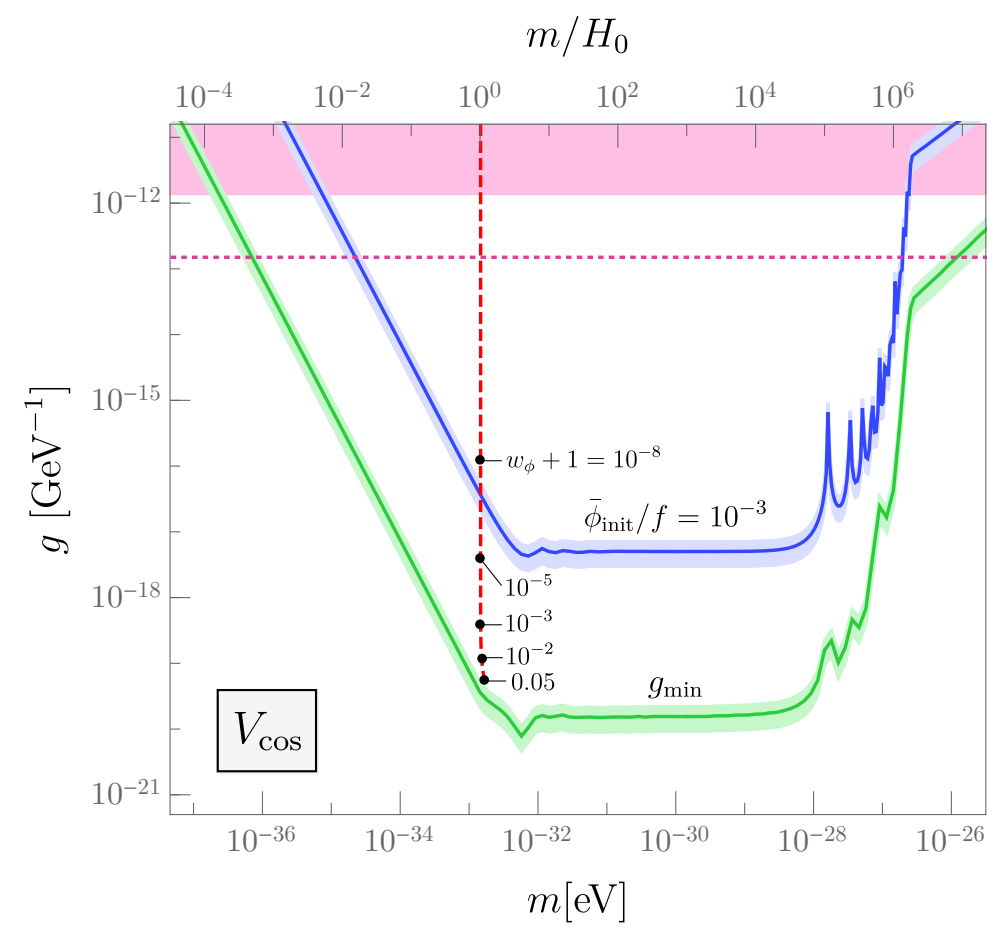

FIG. 2. The ALP-photon coupling constant $g$ inferred by the isotropic birefringence $\bar{\alpha}=0.35 \pm 0.14$ deg versus the ALP mass $m$ for the cosine potential $V_{\cos }(\phi)=m^{2} f^{2}[1-\cos (\phi / f)]$ with $f=M_{\mathrm{pl}}$. The green line corresponds to the case of minimum values of $g$ satisfying $\Omega_{\phi} \leq \Omega_{\phi \text {,max }}$. The blue line adopts the initial condition $\bar{\phi}_{\text {init }} / f=10^{-3}$ and the parameter region above this line requires a fine tuning near $\bar{\phi}_{\text {init }} \sim 0$. The ALP that accounts for all of dark energy by staying on the hilltop of the potential lies on the red dashed line. In this case, we show five different values of $w_{\phi}+1\left(10^{-8}, 10^{-5}, 10^{-3}, 10^{-2}, 0.05\right)$ as the black dots, whose initial conditions $\bar{\phi}_{\text {init }}$ can be found in Fig. 3 Each point on and in the left side of the red dashed line has two corresponding values of $\bar{\phi}_{\text {init }}$ in the regions $0<\bar{\phi}_{\text {init }} / f<\pi / 2$ and $\pi / 2<\bar{\phi}_{\text {init }} / f<\pi$, with the degeneracy at the inflection point $\bar{\phi}_{\text {init }} / f=\pi / 2$ on the green line.

which is tighter than the bound $(29)$.

For $m \gtrsim H_{0}$, the initial field value corresponding to the coupling $g_{\min }$ is mostly in the range $\bar{\phi}_{\text {init }} / f \lesssim 1$, in which regime the potential approximately reduces to $V_{\cos }(\phi) \simeq m^{2} \phi^{2} / 2$. Hence the field excursion from the LSS to today is not different from that for the quadratic potential studied in Sec. [IIA. As we observe in Figs. 11 and 2, the qualitative shapes of the minimum $g$ line in the two ALP potentials are similar to each other for $m \gtrsim H_{0}$. From the theoretical line of $g_{\min }$ in the mass range $H_{0}<m<H_{\mathrm{LSS}}$, we obtain the constraint,

$$
\Omega_{\phi}>1.2 \times 10^{-18}\left(\frac{\bar{\alpha}}{0.35 \mathrm{deg}}\right)^{2}
$$

which is close to the bound (30) derived for the quadratic potential.

In the following, we study the case in which the ALP accounts for all of dark energy, i.e., $\Omega_{\phi}=\Omega_{\Lambda}=0.69$. If the ALP field is near the top of the potential during the epoch of cosmic acceleration, the potential energy is given by $V_{\cos }(\bar{\phi} \simeq \pi f) \simeq 2 m^{2} f^{2}$. When this is responsible for all of dark energy, we require that $2 m^{2} f^{2}=3 M_{\mathrm{pl}}^{2} H_{0}^{2} \Omega_{\Lambda}$ and hence

$$
m=\sqrt{\frac{3 \Omega_{\Lambda}}{2}} \frac{M_{\mathrm{pl}}}{f} H_{0} .
$$

For $f=M_{\mathrm{pl}}$ and $\Omega_{\Lambda}=0.69$, it follows that $m=1.017 H_{0}$. As the initial field value $\bar{\phi}_{\text {init }}$ approaches the inflection point $\bar{\phi}=\pi f / 2, m$ slightly gets larger than the value (36). Moreover, if $\bar{\phi}_{\text {init }}$ is close to $\pi f / 2$, the observational bound $w_{\phi}<-0.95$ tends to be violated due to the large variation of the ALP field.

In Fig. 2, the red dashed line corresponds to the case in which the ALP field acts as all of dark energy. We also show several values of $w_{\phi}+1$ as black dots. The corresponding line and the values of $w_{\phi}+1$ are also plotted on the $\left(m, \pi-\bar{\phi}_{\text {init }} / f\right)$ plane in the right panel of Fig. 3 . As $\bar{\phi}_{\text {init }}$ approaches $\pi f / 2, w_{\phi}$ continues to increase. The observational upper limit $w_{\phi, \max }=0.95$ is reached around $\bar{\phi}_{\text {init }} \simeq 2.2 f$. 

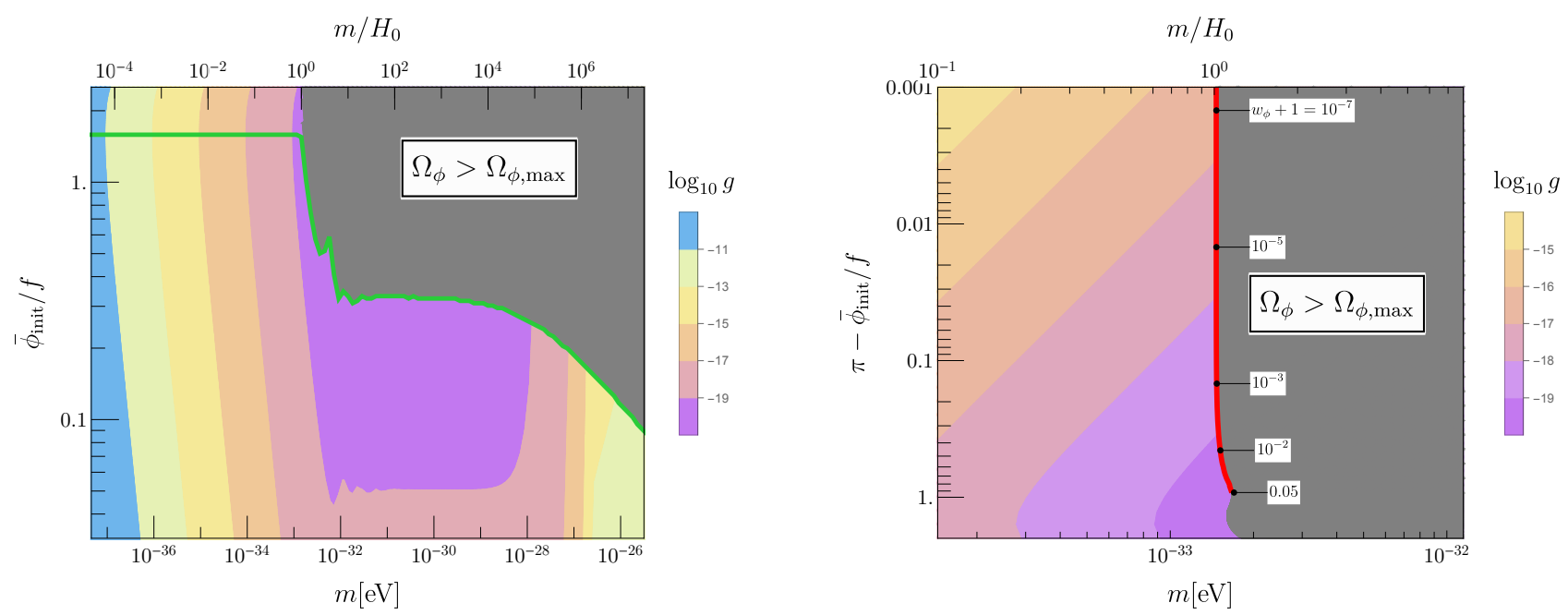

FIG. 3. (Left panel) The contour of the ALP-photon coupling constant $g$ inferred by the isotropic birefringence $\bar{\alpha}=0.35$ deg in the $\left(m, \bar{\phi}_{\text {init }} / f\right)$ plane for the cosine potential (31) with $f=M_{\mathrm{pl}}$. The gray region is excluded by the upper limit of $\Omega_{\phi}$. The green line represents $\bar{\phi}_{\text {init }}$ that gives the minimum value of $g$ for a given mass, and it corresponds to the initial conditions of the green line in Fig. 2 (Right panel) The plot scheme is the same as the left panel except for the vertical axis that changes to focus on the hill-top initial condition, $\bar{\phi}_{\text {init }} \sim \pi f$. The red line represents the ALP parameters accounting for all of dark energy and it corresponds to the red dashed line in Fig. 2. The black dots show five different values of $w_{\phi}+1$.

For $\bar{\phi}_{\text {init }}$ closer to $\pi f$, the deviation of $w_{\phi}$ from -1 decreases toward 0 . As we see in Fig. 2, even the tiny deviation like $w_{\phi}+1=10^{-8}$ predicts the coupling $g$ which is below the current bound of Chandra. If the future axion measurements were to detect the coupling $g$, this can provide a very interesting possibility for probing the tiny deviation of the ALP dark energy equation of state $w_{\phi}$ from -1 . Under the slow-roll approximation where the ALP kinetic energy is subdominant to the potential energy, we have

$$
w_{\phi}=\frac{\dot{\bar{\phi}}^{2} / 2-V}{\dot{\bar{\phi}}^{2} / 2+V} \simeq-1+\frac{\dot{\bar{\phi}}^{2}}{V},
$$

where $V$ is related to $H_{0}$, as $3 M_{\mathrm{pl}}^{2} H_{0}^{2} \simeq\left(1+\Omega_{M} / \Omega_{\phi}\right) V$. Then, the ALP-photon coupling constant can be estimated as

$$
g=\frac{2|\bar{\alpha}|}{|\Delta \phi|} \simeq \frac{2|\bar{\alpha}|}{H_{0}^{-1}|\dot{\bar{\phi}}|}=1.6 \times 10^{-20} \mathrm{GeV}^{-1}\left(\frac{|\bar{\alpha}|}{0.35 \mathrm{deg}}\right)\left(\frac{0.05}{1+w_{\phi}}\right)^{1 / 2}
$$

where we assumed that the field excursion is dominated by the recent contribution, which is true for the thawing models of dark energy. With this equation, the value of $w_{\phi}$ can be estimated on the red dashed line in Fig. 2. Applying the Chandra bound $g<1.4 \times 10^{-12} \mathrm{GeV}^{-1}$ to Eq. (38), it follows that

$$
w_{\phi}+1>6.5 \times 10^{-18}\left(\frac{|\bar{\alpha}|}{0.35 \mathrm{deg}}\right)^{2} .
$$

The field needs to vary at some extent to explain the observed value of isotropic cosmic birefringence. It is interesting to note that the constraint (39) gives a lower bound on $w_{\phi}$ larger than -1 . To our knowledge, this is the first observational lower bound of $w_{\phi}$ forbidding the cosmological constant value -1 .

Finally, we should comment on the fact that one set of $m$ and $g$ does not necessarily determine a unique initial condition $\bar{\phi}_{\text {init }}$. As we see in Fig. 3 , for the mass range $m \lesssim H_{0}$, there are two values of $\bar{\phi}_{\text {init }}$ which correspond to the same $m$ and $g$. One of those initial conditions is in the region $0<\bar{\phi}_{\text {init }} / f<\pi / 2$, whereas the other is in the regime $\pi / 2<\bar{\phi}_{\text {init }} / f<\pi$. In Fig. 2, each set of $m$ and $g$ has two corresponding initial conditions $\bar{\phi}_{\text {init }}$ in the left-side region of the red dashed line and above the green line. On the green line, the two initial conditions are degenerate at the inflection point, $\bar{\phi}_{\text {init }} / f=\pi / 2$.

As the coupling $g$ increases along the red dashed line in Fig. 2, one of $\bar{\phi}_{\text {init }}$ approaches the bottom of potential and the other does the top of potential. As we already mentioned, the initial condition $\bar{\phi}_{\text {init }} / f \ll 1$ requires a fine tuning. 
To obtain the values of $g$ whose orders are the same in the two regimes $\bar{\phi}_{\text {init }} / f \ll 1$ and $\left|\bar{\phi}_{\text {init }} / f-\pi\right| \ll 1$, we need the similar level of fine tuning for the ALP initial conditions. The initial condition with $\left|\bar{\phi}_{\text {init }} / f-\pi\right| \ll 1$ corresponds to the case in which the ALP field can be the source of all of dark energy. In the region above the blue line in Fig. 2 , we require the fine tuning of $\bar{\phi}_{\text {init }}$ in both the two regimes mentioned above.

\section{EARLY DARK ENERGY}

Recently, some scalar-field models were proposed to resolve or alleviate the problem of $H_{0}$ tension between CMB and low-redshift measurements. In these early dark energy (EDE) models, the scalar field is nearly frozen due to the Hubble friction prior to a critical scale factor $a_{c}$ of order $a_{\text {eq }}$, and it plays a role of the cosmological constant with $\tilde{w}_{\phi}$ close to -1 . The additional scalar-field energy density increases the Hubble expansion rate at early times, so the sound horizon around the LSS is reduced by the presence of EDE. Then, the models can be compatible with the Planck data of CMB temperature anisotropies with larger values of $H_{0}$. For $a \geq a_{c}$, the scalar field exhibits damped oscillations with the energy density decaying faster than those of standard matter components. This is possible for the field potential behaving like $V(\phi) \propto \phi^{2 n}$ with $n \geq 2$ around its potential minimum. In this case, the contribution of EDE to the late-time cosmic expansion is negligible.

Previous works identified the parameter space in which the existence of EDE remedies the Hubble tension. In this section, we assume that the scalar field in EDE models is coupled to photon through the coupling $g \phi F_{\mu \nu} \tilde{F}^{\mu \nu} / 4$ and compute how much cosmic birefringence is generated. We will explore two EDE models, (A) higher-order periodic potentials [62, 86] and (B) power-law potentials [63], and obtain the coupling constant for which the observed isotropic birefringence is produced. Therefore, the ALP not only ameliorates the Hubble tension but also explains the observed value of $\bar{\alpha}$ inside the parameter space derived below.

\section{A. Higher-order periodic potentials}

First, we consider the higher-order periodic potentials studied in Refs. [62, 86]:

$$
V_{\cos }^{(n)}(\phi)=m^{2} f^{2}\left[1-\cos \left(\frac{\phi}{f}\right)\right]^{n},
$$

where $f$ is the decay constant and we fix $f=M_{\mathrm{pl}}$ in the following. For $\phi \ll f$, this potential is well approximated by a power-law function $V_{\cos }^{(n)}(\phi) \simeq\left(m^{2} f^{2} / 2^{n}\right)(\phi / f)^{2 n}$. In Ref. 62, the critical redshift $a_{c}$ is defined by

$$
\rho_{\phi}\left(a_{c}\right)=\frac{V_{\cos }^{(n)}\left(\bar{\phi}_{\text {init }}\right)}{2},
$$

which qualitatively indicates that the ALP field begins to oscillate at $a=a_{c}$. The onset of oscillations can be also roughly estimated as $\left|V_{\cos , \bar{\phi} \bar{\phi}}^{(n)}\left(\bar{\phi}_{c}\right)\right| \simeq H_{c}^{2}$, where $\bar{\phi}_{c}$ and $H_{c}$ are the ALP background and the Hubble parameter at $a=a_{c}$, respectively. In the regime $\bar{\phi} \ll f$, this estimation approximately translates to $m^{2}\left(\bar{\phi}_{c} / f\right)^{2(n-1)} \simeq H_{c}^{2}$. For $n \geq 2, H_{c}$ is smaller than $m$. The EDE field starts to oscillate prior to the last scattering epoch, so the mass $m$ should be in the range

$$
m \gg H_{\mathrm{LSS}} \simeq 3.3 \times 10^{-29} \mathrm{eV}
$$

Before and after the transition at $a=a_{c}$, the field equation of state changes from $\tilde{w}_{\phi} \simeq-1$ to $\left\langle\tilde{w}_{\phi}\right\rangle \simeq(n-1) /(n+1)$, where the latter is averaged over oscillations. For $a>a_{c}$ the energy density of $\phi$ decreases as $\rho_{\phi} \propto a^{-6 n /(n+1)}$. This means that, for $n \geq 2$, the contribution of $\rho_{\phi}$ to $H$ becomes negligible compared to nonrelativistic matter.

In Ref. 62, the authors introduced the relative ratio between the density parameters of EDE and total matter at the transition, as $f_{\mathrm{EDE}} \equiv \tilde{\Omega}_{\phi}\left(a_{c}\right) / \tilde{\Omega}_{\text {tot }}\left(a_{c}\right)$. Running a Markov chain Monte Carlo (MCMC) simulation with flat priors on $\log _{10}\left(a_{c}\right), \Omega_{\phi}=\tilde{\Omega}_{\phi}\left(t_{0}\right), \bar{\phi}_{\text {init }}$, and six $\Lambda$ CDM parameters, they obtained the posterior distributions of them. For the likelihood analysis, they used the observational data of SH0ES, Planck, 6dFGS, SDSS, BOSS DR12, and Pantheon. The best-fit value of the Hubble constant was found to be $H_{0}=71.1 \mathrm{~km} / \mathrm{s} / \mathrm{Mpc}$ for $n=2$ and $H_{0}=71.6 \mathrm{~km} / \mathrm{s} / \mathrm{Mpc}$ for $n=3$, so this EDE model can ease the Hubble tension. Here, we use their marginalized 2D posterior distributions of $\log _{10}\left(a_{c}\right)$ and $f_{\mathrm{EDE}}\left(a_{c}\right)$ with $n=2$ and 3 .

To calculate the field excursion $\Delta \bar{\phi}$ and derive the produced isotropic birefringence in this model, one needs to know the mass parameter $m$ and the initial field value $\bar{\phi}_{\text {init }}$. Numerically solving the dynamics of $\bar{\phi}$ for varying parameters, 

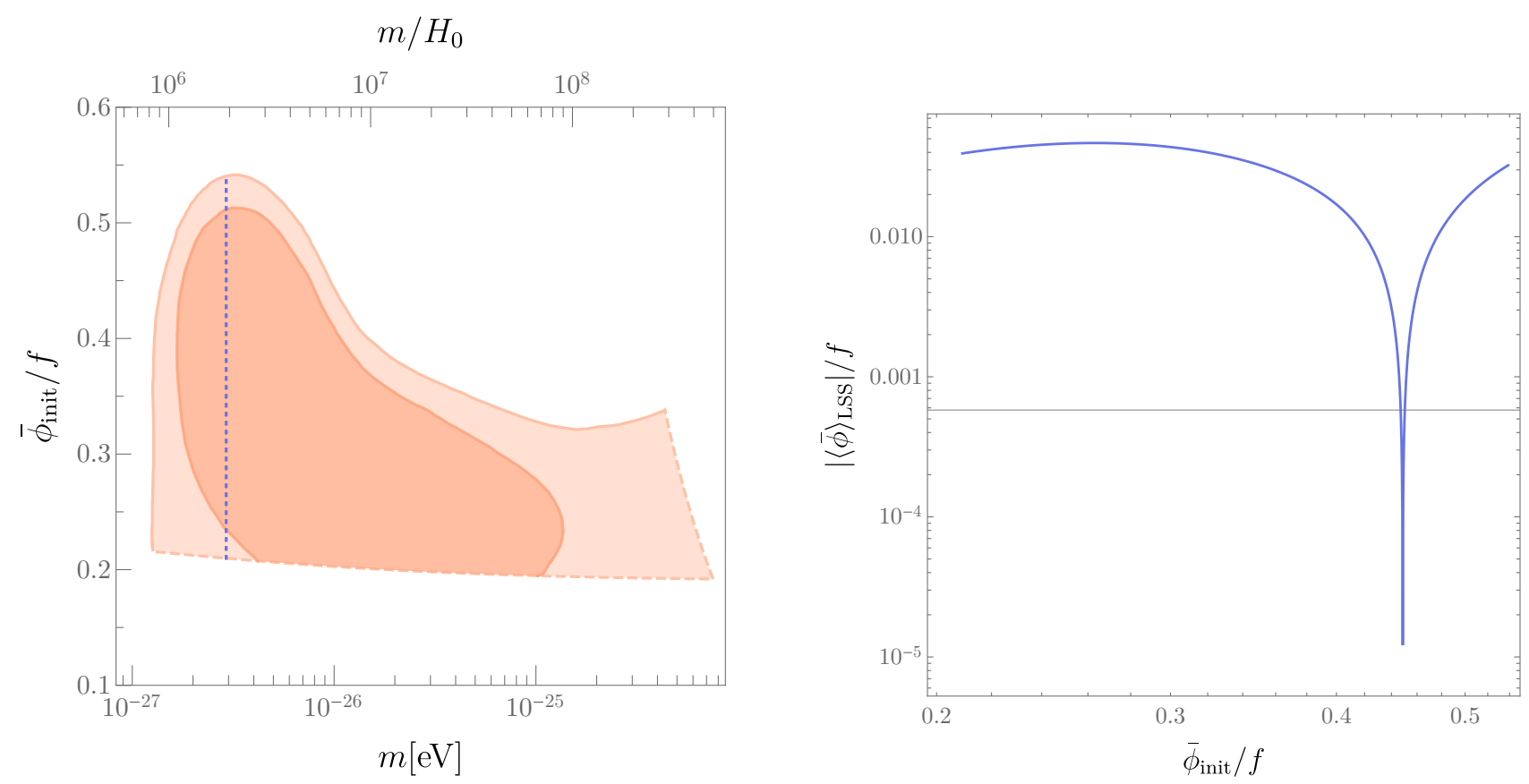

FIG. 4. (Left panel) The $1 \sigma$ and $2 \sigma$ contours of the mass parameter $m$ and the initial EDE field value $\bar{\phi}_{\text {init }} / f$ with the potential $V_{\cos }^{(2)}(\phi)$. We convert the posterior distributions of $a_{c}$ and $f_{\operatorname{EDE}}\left(a_{c}\right)$ in Ref. 62] by (inversely) solving the dynamics of $\bar{\phi}$. A sharp cut-off at the bottom and right edges corresponds to the boundary of the figure in Ref. 62. The blue dotted line denotes $m$ and the domain of $\bar{\phi}_{\text {init }} / f$ with which the right panel is depicted. (Right panel) $\left|\langle\bar{\phi}\rangle_{\text {LSS }}\right| / f$ as a function of $\bar{\phi}_{\text {init }} / f$ within the $2 \sigma$ contour for $m=2.9 \times 10^{-27} \mathrm{eV}$. This value of $m$ corresponds to the best-fit values of $a_{c}$ and $f_{\mathrm{EDE}}\left(a_{c}\right)$. The horizontal gray line denotes the threshold defined in the main text. The region below this threshold includes only $1 \%$ of the $\bar{\phi}_{\text {init }} / f$ domain and it is less likely to have such an initial value, which accidentally suppresses $\left|\langle\bar{\phi}\rangle_{\text {LSS }}\right| / f$.

we convert the posterior distributions of $a_{c}$ and $f_{\mathrm{EDE}}\left(a_{c}\right)$ in Ref. 62 to the distribution of $m$ and $\bar{\phi}_{\text {init }}$. The result is shown in the left panel of Fig. 4. In Ref. 62 the posterior distribution was presented only for $f_{\mathrm{EDE}}\left(a_{c}\right)>0.01$, presumably because $\phi$ is not effective to reduce the Hubble tension for a smaller energy fraction. Following Ref. [62, we disregard the distribution for $f_{\mathrm{EDE}}\left(a_{c}\right)<0.01$, and hence the contour in the left panel of Fig. 4 has a sharp cut-off at its bottom edge.

For the derivation of $\bar{\alpha}$ in Eq. (14), we also compute $\langle\bar{\phi}\rangle_{\text {LSS }}$ by integrating Eq. 19.). As we already discussed in Sec. II. the suppression of $\langle\bar{\phi}\rangle_{\text {LSS }}$ by the fast oscillation of $\bar{\phi}$ around the LSS also occurs for the present potential with the mass scale $\sqrt{42}$. To illustrate this effect, we show the averaged value $\langle\bar{\phi}\rangle_{\text {LSS }}$ as a function of the initial value $\bar{\phi}_{\text {init }}$ for $m=2.9 \times 10^{-27} \mathrm{eV}$ in the right panel of Fig. 4. Since $\langle\bar{\phi}\rangle_{\mathrm{LSS}}$ is obtained by convoluting the oscillating field $\bar{\phi}$ with the positive visibility function, its sign can be positive or negative depending on the phase of $\bar{\phi}$. When we change $\bar{\phi}_{\text {init }} / f$ continuously, the oscillation phase slides and the sign of $\langle\bar{\phi}\rangle_{\text {LSS }}$ flips at certain values of $\bar{\phi}_{\text {init }} / f$. Therefore $\left|\langle\bar{\phi}\rangle_{\text {LSS }}\right|$ passes through zero for these initial conditions, which appear as a sharp dip in the right panel of Fig. 4. For such $\bar{\phi}_{\text {init }}, \Delta \bar{\phi}$ is also subject to suppression and one apparently needs a large $g$ to account for the observed $\bar{\alpha}$. Nevertheless, it is less likely to have such specifically small values of $\left|\langle\bar{\phi}\rangle_{\text {LSS }}\right|$ with high precision by chance. To quantify this fine tuning, we introduce a threshold of $\left|\langle\bar{\phi}\rangle_{\text {LSS }}\right|$ above which $99 \%$ of the interval of $\bar{\phi}_{\text {init }} / f$ within the $1 \sigma$ or $2 \sigma$ contour is included for a given $m$. This threshold is shown as a solid horizontal line in the right panel of Fig. 4 and the probability to realize $\left|\langle\bar{\phi}\rangle_{\text {LSS }}\right|$ less than this threshold is smaller than $1 \%$.

In Figs. 5 and 6 , we plot the $1 \sigma$ and $2 \sigma$ contours in the $(m, g)$ plane which explain the observed $\bar{\alpha}$ and also reduce the Hubble tension for the potential $\sqrt{40}$ with $n=2$ and 3. Since $\left|\langle\bar{\phi}\rangle_{\text {LSS }}\right|$ can vanish for some special initial conditions of $\bar{\phi}$, the contour of $m$ and $\bar{\phi}_{\text {init }} / f$ in the left panel of Fig. 4 does not put an upper bound on $g$ in a rigorous sense. Thus we show the $1 \sigma$ and $2 \sigma$ regions enclosed by the lower bound from the EDE contour (solid line) and the sketchy upper bound inferred by the fine-tuning threshold discussed above (dashed line). Although there is a small chance to have $g$ larger than the threshold, the probability rapidly decreases as $g$ increases. This feature is expressed by the gradation of the light orange color above the contour.

The left panels of Figs. 5 and 6 do not take into account the uncertainty of $\bar{\alpha}$, but we merely use its best-fit value, $\bar{\alpha}=0.35$ deg. It is tricky to combine the uncertainties of the EDE model parameters and that of $\bar{\alpha}$, because we do not have the complete information of their posterior distributions. To present conservative contour plots, we sweep from 

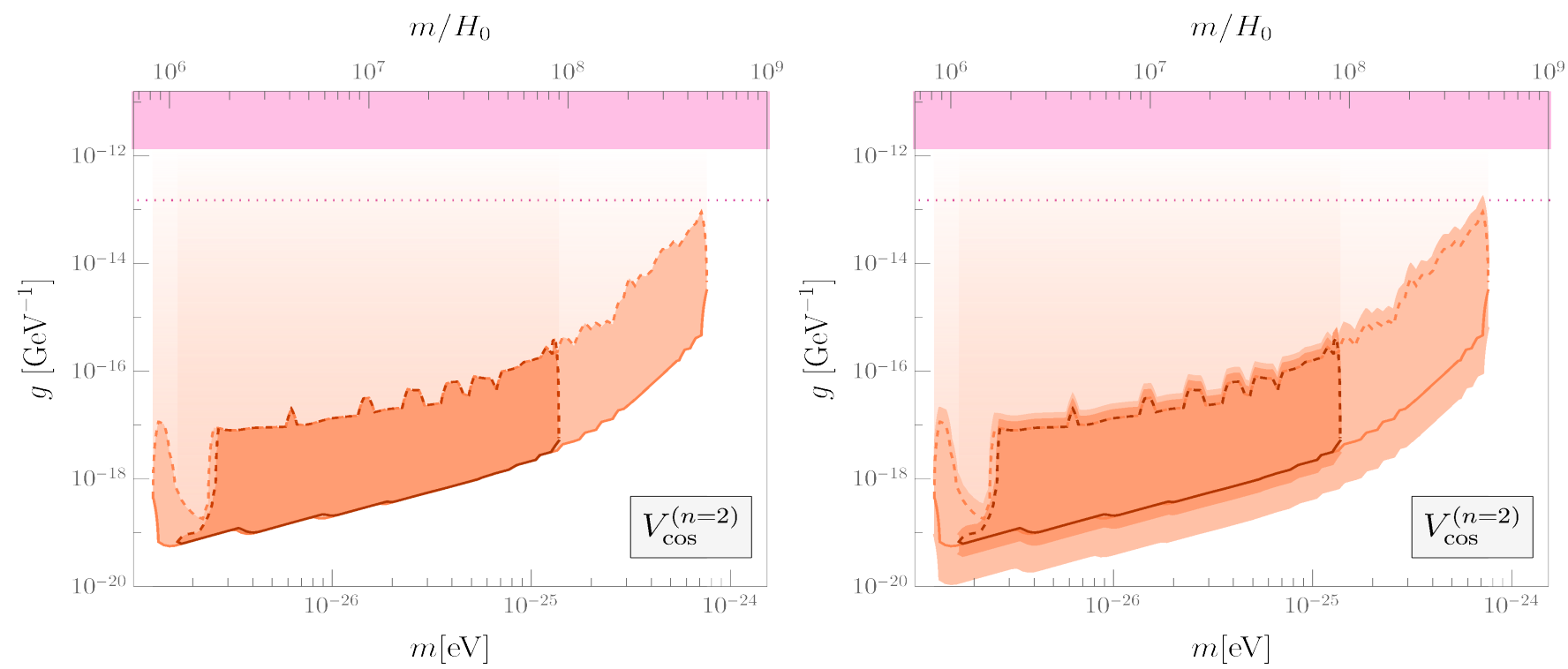

FIG. 5. (Left panel) The orange shaded regions denote the $1 \sigma$ (dark) and $2 \sigma$ (light) contours in the ( $m, g$ ) plane for the EDE model with the higher-order periodic potential $V_{\cos }^{(n=2)}(\phi)$. We fix the cosmic birefringence to be the observed best-fit value $\bar{\alpha}=0.35 \mathrm{deg}$. The dashed lines denote the fine-tuning threshold below which $99 \%$ of the initial value $\bar{\phi}_{\text {init }}$ is included. As $g$ goes higher than the threshold line, it becomes less likely to realize, although it is not rigorously excluded. This feature is expressed by the gradation of the light orange color above the contour. The pink shaded region is constrained by Chandra. The pink dotted line is the projected sensitivity of Athena. (Right panel) The dark and light orange regions are the $1 \sigma$ and $2 \sigma$ EDE model contours extended by the $1 \sigma$ and $2 \sigma$ uncertainties of the observed $\bar{\alpha}$ (i.e., $\delta \bar{\alpha}=0.14 \mathrm{deg}$ and $\delta \bar{\alpha}=0.28$ deg), respectively. The solid, dashed, and dotted lines are all the same as those in the left panel.
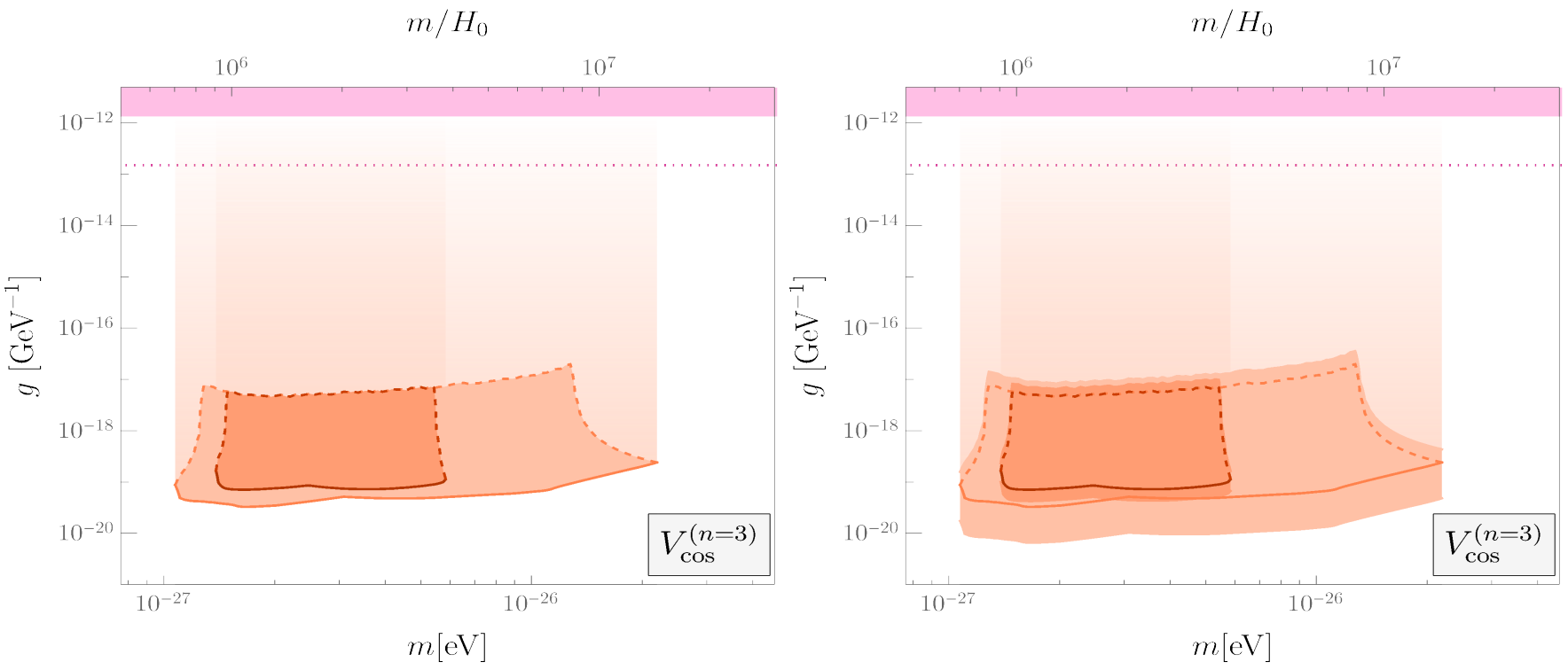

FIG. 6. The plot scheme is the same as Fig. 5 , while the EDE model potential is replaced by $V_{\cos }^{(n=3)}(\phi)$.

$\bar{\alpha}=(0.35-0.14) \mathrm{deg}$ to $(0.35+0.14) \mathrm{deg}$ for the $1 \sigma$ EDE contour and from $\bar{\alpha}=(0.35-0.28) \operatorname{deg}$ to $(0.35+0.28) \mathrm{deg}$ for the $2 \sigma$ EDE contour in the right panels of Figs. 5 and 6 . This treatment does not follow the general rule of error propagation, so it can overestimate the uncertainty to some extent. Thus one should consider the contours in these right panels as crude but conservative constraints which do not exactly correspond to $1 \sigma$ or $2 \sigma$. 

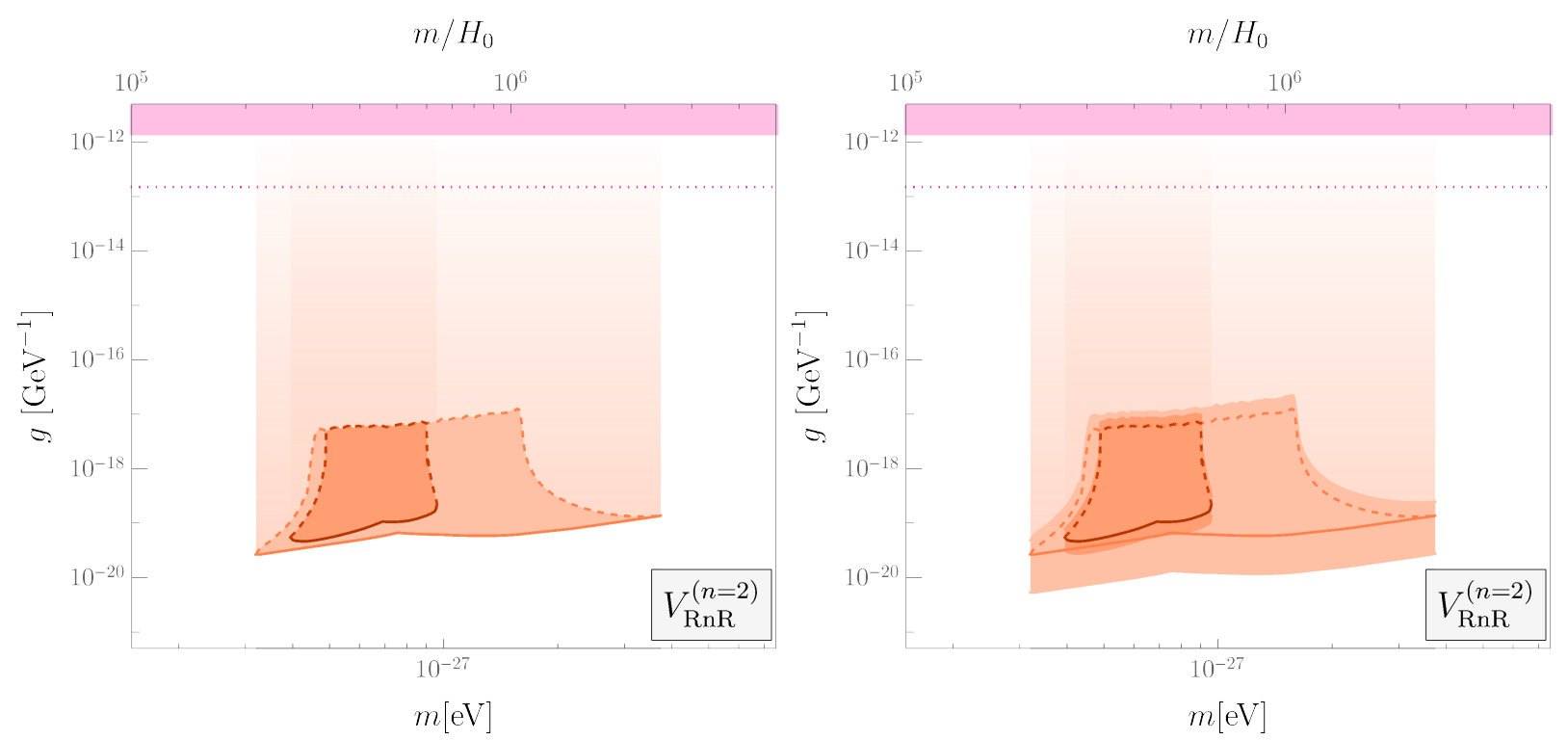

FIG. 7. The plot scheme is the same as Figs. 5 and 6, while the rock ' $\mathrm{n}$ ' roll model with $n=2$ is employed.

\section{B. Rock ' $n$ ' roll model}

As another interesting proposal of the EDE potential, we consider the rock 'n' roll model studied in Ref. 63]:

$$
V_{\mathrm{RnR}}^{(n)}(\phi)=V_{0}\left(\frac{\phi}{M_{\mathrm{Pl}}}\right)^{2 n}=\frac{m^{2} M_{\mathrm{Pl}}^{2}}{2^{n}}\left(\frac{\phi}{M_{\mathrm{Pl}}}\right)^{2 n},
$$

where $V_{0}, n$, and $m$ are constants. Here, we defined the mass $m$ such that the higher-order periodic potential 40) with $f=M_{\mathrm{pl}}$ asymptotes to 43 in the vicinity of the origin, $\phi=0$. For $n \geq 2$, a scalar field with this potential can work as the source of EDE.

In Ref. 63], the authors introduced two parameters, the critical redshift $a_{c}$ defined by $V_{\mathrm{RnR}, \bar{\phi} \bar{\phi}}^{(n)}\left(\bar{\phi}_{c}\right)=9 H^{2}\left(a_{c}\right)$, and the energy fraction of the EDE potential to the total energy at $a_{c}, f_{\phi} \equiv V_{\mathrm{RnR}}^{(n)}\left(a_{c}\right) / \rho_{\mathrm{tot}}\left(a_{c}\right)$. These parameters are defined in a slightly different way in comparison to those for the potential 40 . Using the datasets of SH0ES, Planck, 6dFGS, SDSS, BOSS DR12, and Pantheon and running a MCMC simulation with flat priors on $\log _{10}\left(a_{c}\right), f_{\phi}$, and six $\Lambda \mathrm{CDM}$ parameters, they obtained the posterior distributions of $a_{c}$ and $f_{\phi}$. For $n=2$ the best-fit value of the Hubble constant was found to be $H_{0}=70.5 \mathrm{~km} / \mathrm{s} / \mathrm{Mpc}$, so this EDE model also reduces the Hubble tension. Here, we use their marginalized 2D posterior distributions of $\log _{10}\left(a_{c}\right)$ and $f_{\phi}$ with $n=2$.

Analogous to the discussion in Sec. IV A we translate the $\left(a_{c}, f_{\phi}\right)$ contour derived in Ref. [63] to the distribution of $m$ and $\bar{\phi}_{\text {init }}$ by solving the dynamics of $\phi$. In this step, we disregard the region of $\left(a_{c}, f_{\phi}\right)$ with $f_{\phi}<0.01$ for the same reason explained in Sec. IV A. Our results are shown in Fig. 7. Compared to Fig. 5 whose potential $V_{\text {cos }}^{(2)}(\phi)$ asymptotes $V_{\mathrm{RnR}}^{(2)}(\phi)$ around the origin, the contour in Fig. 7 favors a lower mass $m$, with the shrink of an allowed region of $m$. Note that the treatments of the ALP dynamics are not identical to each other between Ref. [62] and [63], which might lead to the deviation of the results in addition to the intrinsic model difference.

\section{Common features in two EDE models}

We discuss some common results in the two EDE models presented in Secs. IVA and IVB The lower bounds on $g$ consistent with the observed value of $\bar{\alpha}$ can be seen in Figs. 5 7 7. A careful reader may notice that these lower limits are even smaller than those of the simple models obtained in Sec. III for similar values of $m$ (see the green lines in Figs. 1 and 2). This is because the suppression by the time averaging in Eq. (12) is less significant in the EDE models. The effective mass squared of the EDE field around $\phi=0$ is given by $V_{, \phi \phi}(\phi) \sim m^{2}(\phi / f)^{2(n-1)}$, so it decreases as the amplitude of $\bar{\phi}$ decays during the oscillation. Hence the EDE field oscillates more slowly in comparison to the scalar field with a constant mass $m$. Unlike the models studied in Sec. III the values of $\left|\langle\bar{\phi}\rangle_{\mathrm{LSS}}\right|$ are typically larger than 
$\left|\bar{\phi}_{\text {obs }}\right|$ for the constrained model parameters shown in Figs. 5.7 (apart from the $1 \%$ of the dip of $\left|\langle\bar{\phi}\rangle_{\text {LSS }}\right|$ seen in the right panel of Fig. 4).

One also observes in Figs. 5.7 that the coupling constant $g$ accounting for the observed $\bar{\alpha}$ and reducing the Hubble tension is much smaller than the current Chandra bound $g<1.4 \times 10^{-12} \mathrm{GeV}^{-1}$. Unfortunately, it looks difficult for the future observation by Athena to detect a signal of the axion-photon coupling. It is worth considering how to confirm our scenario where the scalar field in the EDE models with the photon coupling produces the isotropic birefringence. We will discuss this issue in Sec. V

Finally, it is interesting to note that the contours in Figs. 5 |7 are centered at

$$
g \sim 10^{-18} \mathrm{GeV}^{-1} .
$$

This means that $g f$ is a dimensionless number of order unity. We can rewrite Eq. [14, as

$$
g f=\frac{2 \bar{\alpha}}{\Delta \bar{\phi} / f} \simeq \frac{2 \bar{\alpha}}{\langle\bar{\phi}\rangle_{\mathrm{LSS}} / f},
$$

where we ignored the sub-leading contribution from $\bar{\phi}_{\text {obs }}$. The observed value of isotropic birefringence is a small number in radians, $\bar{\alpha} \simeq 6 \times 10^{-3}$, and $\langle\bar{\phi}\rangle_{\text {LSS }}$ is subject to suppression by the time averaging. Therefore the fact that $g f=\mathcal{O}(1)$ appears to be quite non-trivial and it may imply something about Planck scale physics. Although we fix $f=M_{\mathrm{pl}}$ in this paper, the same exercises can be done for other values of $f$.

\section{DISCUSSION}

In this section, we discuss the contributions to the CMB birefringence from the ALP fluctuation, namely $\delta \phi_{\text {LSS }}$ and $\delta \phi_{\text {obs }}$ in Eq. (5), which have been ignored so far. The ALP fluctuation has two possible origins: One is the quantum perturbation produced during inflation $\delta \phi^{(\mathrm{inf})}$, and the other is the sourced perturbation induced by the adiabatic mode during the background ALP evolution $\delta \phi^{(\mathrm{src})}$.

Let us mainly consider the massive ALP potential given by Eq. (20). Since we are interested in the mass scale $m$ much smaller than the inflationary Hubble parameter $H_{\text {inf }}$, the amplitude of $\delta \phi^{(\text {inf })}$ is of the order $\sim H_{\text {inf }} /(2 \pi)$, when the primordial perturbation is produced around the Hubble radius crossing. However, the evolution of $\delta \phi^{\text {(src) }}$ as well as $\delta \phi^{(\mathrm{inf})}$ highly depends on the models and their parameters. It requires a dedicated investigation to calculate their contributions to the birefringence in the full range of $m$, which is beyond the scope of this paper.

Instead, we split the parameter range into three parts, (i) the light region: $m \lesssim H_{0}$, (ii) the heavy region: $m \gtrsim H_{\text {LSS }}$, and (iii) the intermediate region: $H_{0} \lesssim m \lesssim H_{\mathrm{LSS}}$, and briefly explore them in order. We give simple estimations of the fluctuations and make comments on their potential effects on the cosmic birefringence.

In the mass region $m \lesssim H_{0}$, the sourced perturbation is not relevant to the observed $\bar{\alpha}$ because the source term for $\delta \phi$ is proportional to $\dot{\bar{\phi}} \propto m^{2}$ and thus negligible. Then it is straightforward to track the evolution of $\delta \phi$ originating from $\delta \phi^{(\mathrm{inf})}$. In doing so, we introduce the tensor-to-scalar ratio $r=2 H_{\mathrm{inf}}^{2} /\left(\pi M_{\mathrm{pl}}^{2} P_{\zeta}\right)$, where $P_{\zeta}$ is the scalar power spectrum generated during inflation. The contributions of perturbations at present and at the LSS to the birefringence can be computed, respectively, as 72

$$
\begin{aligned}
\frac{\left|\bar{\alpha}_{\delta \phi}\right|}{0.35 \mathrm{deg}} & =\left(\frac{g}{3.0 \times 10^{-15} \mathrm{GeV}^{-1}}\right)\left(\frac{r}{0.06}\right)^{1 / 2}, \\
\frac{A_{\alpha}}{0.033 \mathrm{deg}^{2}} & =\left(\frac{g}{1.1 \times 10^{-15} \mathrm{GeV}^{-1}}\right)^{2}\left(\frac{r}{0.06}\right),
\end{aligned}
$$

where $A_{\alpha}=L(L+1) C_{L}^{\alpha \alpha} /(2 \pi)$ characterizes the anisotropic birefringence with $C_{L}^{\alpha \alpha}$ being the angular power spectrum of $\alpha(\hat{\boldsymbol{n}})$, and we used its current upper bound $A_{\alpha} \leq 0.033 \mathrm{deg}^{2}$ [75, 76] as a reference value. Here $\bar{\alpha}_{\delta \phi}$ denotes $\bar{\alpha}$ contributed by $\delta \phi_{\text {obs }}$. Eliminating $g$ and $r$ from the above equations, we obtain the relation between $\bar{\alpha}_{\delta \phi}$ and $A_{\alpha}$, as

$$
\left|\bar{\alpha}_{\delta \phi}\right|=0.13 \operatorname{deg} \times\left(\frac{A_{\alpha}}{0.033 \operatorname{deg}^{2}}\right)^{1 / 2} .
$$

It implies that, since $\delta \phi_{\mathrm{LSS}}$ and $\delta \phi_{\mathrm{obs}}$ are connected to each other, the upper bound on the anisotropic birefringence also puts a constraint on the isotropic birefringence which is generated by the ALP fluctuation. Therefore, we expect that $\delta \phi_{\text {obs }}$ gives only a subdominant contribution to the observed $\bar{\alpha}$ for $m \lesssim H_{0}$. Nonetheless, $\delta \phi_{\text {obs }}$ has a stochastic nature, in that Eq. (46) is evaluated by its root mean square and hence there is a chance that $\delta \phi_{\text {obs }}$ gives a larger contribution. An interested reader may refer to Ref. [72] for more details on $\delta \phi_{\text {obs. }}$. 
In the mass region $m \gtrsim H_{\mathrm{LSS}}$, the ALP begins to oscillate before the last scattering epoch. In the simple ALP models, $\langle\bar{\phi}\rangle_{\text {LSS }}$ is exponentially suppressed by the averaging $\sqrt{12}$, and the amplitude of $\bar{\phi}$ has been damped by today. In the EDE models, on the other hand, the suppression effect is less significant due to the field-dependent effective mass decaying in time. This is expected to be true for $\delta \phi_{\text {LSS }}$ as well. In fact, Ref. [73 computed the effective sourced fluctuation $\left\langle\delta \phi_{\mathrm{LSS}}^{(\mathrm{src})}\right\rangle$ for the potential 40 with $n=2,3$ and $f=M_{\mathrm{pl}}$, and found that idealized future CMB experiments could detect the anisotropic birefringence down to $g \sim 10^{-17} \mathrm{GeV}^{-1}$. This value roughly corresponds to the upper part of the contours in Figs. 5 and 6. Therefore, future observations may confirm the prediction of the EDE models and further investigation is awaiting to be done.

For the higher mass region $m \gtrsim 10^{-26} \mathrm{eV}$, the ALP behavior becomes closer to the major dark matter component and its clustering may be relevant in the simple ALP models. Considering the local dark matter density much higher than the averaged one, it may be possible that the ALP clustering boosts its fluctuation and makes $\delta \phi_{\text {obs }}$ significantly contribute to the isotropic birefringence. To give a crude estimate, we assume that the ALP follows the normal dark matter distribution. Then, by scaling the dark matter density, one finds the local ALP amplitude, as

$$
\phi_{\text {local }} \sim \sqrt{\frac{2 \rho_{\text {local }} \Omega_{\phi}}{m^{2} \Omega_{c}}}=2.5 \times 10^{11} \mathrm{GeV}\left(\frac{\Omega_{\phi}}{10^{-2} \Omega_{c}}\right)^{1 / 2}\left(\frac{m}{10^{-24} \mathrm{eV}}\right)^{-1}
$$

where $\Omega_{c}$ denotes the energy fraction of all dark matter, and the local dark matter density $\rho_{\text {local }}$ is set to be $0.4 \mathrm{GeV} / \mathrm{cm}^{3} . \phi_{\text {local }}$ contributes to the isotropic birefringence through the $\delta \phi_{\text {obs }}$ term in Eq. (5) and the corresponding coupling constant is given by

$$
g_{\delta \phi_{\mathrm{obs}}}=\frac{2 \bar{\alpha}}{\phi_{\text {local }}} \sim 5 \times 10^{-14} \mathrm{GeV}^{-1}\left(\frac{\bar{\alpha}}{0.35 \mathrm{deg}}\right)\left(\frac{\Omega_{\phi}}{10^{-2} \Omega_{c}}\right)^{-1 / 2}\left(\frac{m}{10^{-24} \mathrm{eV}}\right) .
$$

This value is much smaller than the naive extrapolation of Figs. 1 and 2 to $m=10^{-24} \mathrm{eV}$ as well as the current upper bound. Thus, such a heavy ALP at the observer might be able to dominate the observed isotropic birefringence in the simple models. However, it should be noted that, in the above crude estimate, we ignore the de Broglie wavelength of the ALP reaching or exceeding the galactic scale, the quantum pressure preventing its clustering on a smaller scale, and the oscillation of the ALP field whose period is $\sim 100\left(\mathrm{~m} / 10^{-24} \mathrm{eV}\right)^{-1} \mathrm{yr}$. We leave the evaluation of the contribution from the local ALP density to the birefringence for future work.

Finally, the intermediate region $H_{0} \lesssim m \lesssim H_{\text {LSS }}$ has not been studied well in the literature. The ALP oscillation starts after the decoupling of CMB photons and the source effect is negligible before that. Eq. (47) is applicable for $\delta \phi_{\mathrm{LSS}}$, while $\delta \phi_{\mathrm{obs}}^{(\mathrm{inf})}$ is more suppressed than that estimated by Eq. 46) due to the damped oscillation. The ALP clustering may be negligible due to its extremely large de Broglie wavelength. It would be interesting to investigate the sourced fluctuation $\delta \phi_{\text {obs }}^{\text {(src) }}$ in this mass region in the simple ALP models.

\section{CONCLUSION}

Cosmic birefringence is a powerful tool to investigate the properties of ultra-light ALPs. The recent analysis based on the Planck 2018 polarization data reported the rotation angle of CMB polarization $\bar{\alpha}=0.35 \pm 0.14$ deg, excluding the null hypothesis at $99.2 \%$ CL. The isotropic birefringence indicates the difference of ALP field values between the last scattering and the detection of CMB photon caused by the dynamics of the background ALP field. Thus, the detected signal opened up a new window for the study of dark energy and early dark energy.

In this paper, we studied the possible origins of isotropic birefringence signal by solving the ALP background dynamics for various potentials $V(\phi)$. The ALP effective mass, which is associated with the second derivative $V_{, \phi \phi}(\phi)$, is a crucial quantity to characterize the epoch at which the initially slow-rolling field starts to oscillate around the potential minimum. The field dynamics translates to the rotation angle through the relation $\bar{\alpha}=g \Delta \bar{\phi} / 2$ as Eq. (14). We included the effect of finite thickness of the LSS, which suppresses the net rotation angle if the ALP field begins to oscillate before the decoupling of CMB photons. Finally, we determined the required value of the ALP-photon coupling constant, $g$, on each parameter to explain the observed isotropic birefringence.

In Sec. III we investigated the simple ALP models with two potentials: the quadratic potential, $V_{\text {mass }}(\phi)=m^{2} \phi^{2} / 2$ of Eq. 201, and the cosine-type potential, $V_{\cos }(\phi)=m^{2} f^{2}[1-\cos (\phi / f)]$ of Eq. (31). Figs. 1 and 2 show the axionphoton coupling inferred from the observed isotropic rotation against the ALP mass. We found that the inferred values of $g$ can be smallest for $H_{0} \lesssim m \lesssim H_{\mathrm{LSS}}$, while in other mass ranges the smaller field variation $\Delta \bar{\phi}$ leads to the larger $g$. Using the dependence of $g$ on $m$ and $\Omega_{\phi}$, we put lower bounds on $g, m$, and $\Omega_{\phi}$ for both potentials. Moreover, we studied the possibility of ALP as dark energy and derived the relation between the ALP-photon coupling and the field equation of state of $w_{\phi}$, in Eq. (38). Using this relation, we put the lower bound of $w_{\phi}$ as $w_{\phi}+1 \gtrsim 6.5 \times 10^{-18}$ 
in Eq. (39), which is a quite surprising result since such a small deviation of $w_{\phi}$ from -1 is far out of reach of the previous constraints derived by standard distance measurements.

In Sec. [V] we studied the signature of ALP-photon coupling in cosmic birefringence for two typical models of EDE: the higher-order periodic potential, $V_{\cos }^{(n)}(\phi)=m^{2} f^{2}[1-\cos (\phi / f)]^{n}$ of Eq. 440$)$, and the rock 'n' roll model, $V_{\mathrm{RnR}}^{(n)}(\phi)=m^{2} M_{\mathrm{pl}}^{2}\left(\phi / M_{\mathrm{pl}}\right)^{2 n} / 2^{n}$ of Eq. 43. The EDE is motivated to alleviate the observational tension of today's Hubble constant between the CMB 4 ] and local astrophysical observations at low redshifts [40 45. Since the successful EDE scenarios require the very limited ranges of ALP mass and initial conditions, we can rigorously estimate the ALP-photon coupling based on the EDE models. In Figs. 5, 6, and 7, we showed the axion-photon coupling inferred from the observed isotropic rotation with $1 \sigma$ and $2 \sigma$ parameter regions for the successful EDE scenarios. The upper bound of $g$ is plotted by requiring the $1 \%$ fine tuning of the ALP initial condition, and the more fine-tuned the initial condition is, the larger $g$ is. We found that the observed isotropic birefringence typically requires $g \sim 10^{-18} \mathrm{GeV}$ for the EDE models in Eq. (44), and it results in $g f=\mathcal{O}(1)$ for $f=M_{\mathrm{pl}}$, which is a non-trivial coincidence. In this paper, we focused on the higher-order periodic potential with $f=M_{\mathrm{pl}}$ and leave the dependence on $f$ for future work.

In Sec. $\mathrm{V}$ ] we commented on other possible sources of isotropic birefringence by ALP. The isotropic birefringence can be induced not only by the background dynamics but also by the fluctuation at the observer's position, $\delta \phi_{\text {obs }}$. To estimate $\delta \phi_{\text {obs }}$, we divided the ALP mass range into three different regions, (i) the light region: $m \lesssim H_{0}$, (ii) the heavy region: $m \gtrsim H_{\mathrm{LSS}}$, and (iii) the intermediate region: $H_{0} \lesssim m \lesssim H_{\mathrm{LSS}}$, and briefly explore each of them. In the region (i), the ALP fluctuation is mostly given by the primordial perturbation during inflation. We found that $\delta \phi_{\text {obs }}$ is unlikely to explain the observed isotropic birefringence since the contribution of such ALP fluctuations is constrained by the observation of anisotropic birefringence. In the region (ii), the ALP fluctuation may be mainly produced by the gravitational growth around galaxies. We roughly estimated the ALP field value as the sub-component of the local dark matter density, and found that the observed signal might be explained by the ALP with $g \sim 10^{-14} \mathrm{GeV}^{-1}$, $m \sim 10^{-24} \mathrm{eV}$, and $\Omega_{\phi} \sim 10^{-2} \Omega_{c}$ in Eq. (50). We leave the region (iii) for future work since the de Broglie wavelength of ALP is larger than the size of galaxies and the structure formation of ALP is unclear in such a mass region.

\section{ACKNOWLEDGEMENTS}

We would like to thank Yuto Minami and Masahiro Kawasaki for fruitful discussions and productive comments. This work is supported by the Grant-in-Aid for Scientific Research Fund of the JSPS Nos. 18K13537 (T. F.), 20J20248 (K. M.), 19J21974 (H. N.), and 19K03854 (S. T.). K. M. is supported by World Premier International Research Center Initiative (WPI Initiative), MEXT, Japan and the Program of Excellence in Photon Science. H. N. is supported by Advanced Leading Graduate Course for Photon Science.

[1] D. Spergel et al. (WMAP), Astrophys. J. Suppl. 148, 175 (2003), arXiv:astro-ph/0302209

[2] J. Weiland, N. Odegard, R. Hill, E. Wollack, G. Hinshaw, M. Greason, N. Jarosik, L. Page, C. Bennett, J. Dunkley, et al., The Astrophysical Journal Supplement Series 192, 19 (2011).

[3] P. Ade et al. (Planck), Astron. Astrophys. 571, A16 (2014), arXiv:1303.5076 [astro-ph.CO]

[4] N. Aghanim et al. (Planck), Astron. Astrophys. 641, A6 (2020), arXiv:1807.06209 [astro-ph.CO].

[5] Y. Minami and E. Komatsu, Phys. Rev. Lett. 125, 221301 (2020), arXiv:2011.11254 [astro-ph.CO].

[6] S. M. Carroll, Phys. Rev. Lett. 81, 3067 (1998), arXiv:astro-ph/9806099.

[7] A. Lue, L.-M. Wang, and M. Kamionkowski, Phys. Rev. Lett. 83, 1506 (1999), arXiv:astro-ph/9812088

[8] M. Pospelov, A. Ritz, C. Skordis, A. Ritz, and C. Skordis, Phys. Rev. Lett. 103, 051302 (2009), arXiv:0808.0673 [astro-ph]

[9] F. Finelli and M. Galaverni, Phys. Rev. D 79, 063002 (2009), arXiv:0802.4210 [astro-ph].

[10] S. Lee, G.-C. Liu, and K.-W. Ng, Phys. Rev. D 89, 063010 (2014), arXiv:1307.6298 [astro-ph.CO].

[11] W. Zhao and M. Li, Phys. Rev. D 89, 103518 (2014), arXiv:1403.3997 [astro-ph.CO],

[12] S. Lee, G.-C. Liu, and K.-W. Ng, The Universe 4, 29 (2016), arXiv:1912.12903 [astro-ph.CO].

[13] G.-C. Liu and K.-W. Ng, Phys. Dark Univ. 16, 22 (2017), arXiv:1612.02104 [astro-ph.CO]

[14] R. Peccei and H. R. Quinn, Phys. Rev. Lett. 38, 1440 (1977).

[15] J. E. Kim, Phys. Rev. Lett. 43, 103 (1979).

[16] M. A. Shifman, A. Vainshtein, and V. I. Zakharov, Nucl. Phys. B 166, 493 (1980)

[17] D. J. E. Marsh, Phys. Rept. 643, 1 (2016), arXiv:1510.07633 [astro-ph.CO].

[18] Y. Gong, X. Chen, and H. Feng, Phys. Rev. Lett. 118, 061101 (2017), arXiv:1612.05697 [astro-ph.HE].

[19] P. Svrcek and E. Witten, JHEP 06, 051 (2006), arXiv:hep-th/0605206

[20] A. Arvanitaki, S. Dimopoulos, S. Dubovsky, N. Kaloper, and J. March-Russell, Phys. Rev. D 81, 123530 (2010) arXiv:0905.4720 [hep-th].

[21] B. Feng, H. Li, M. Li, and X. Zhang, Phys. Lett. B620, 27 (2005), arXiv:hep-ph/0406269 [hep-ph] 
[22] B. Feng, M. Li, J.-Q. Xia, X. Chen, and X. Zhang, Phys. Rev. Lett. 96, 221302 (2006), arXiv:astro-ph/0601095 [astro-ph]

[23] G.-C. Liu, S. Lee, and K.-W. Ng, Phys. Rev. Lett. 97, 161303 (2006), arXiv:astro-ph/0606248 [astro-ph]

[24] M. Fukugita and T. Yanagida, (1994).

[25] J. A. Frieman, C. T. Hill, A. Stebbins, and I. Waga, Phys. Rev. Lett. 75, 2077 (1995), arXiv:astro-ph/9505060

[26] J. E. Kim, JHEP 05, 022 (1999), arXiv:hep-ph/9811509

[27] J. E. Kim, JHEP 06, 016 (2000), arXiv:hep-ph/9907528

[28] K. Choi, Phys. Rev. D 62, 043509 (2000), arXiv:hep-ph/9902292

[29] Y. Nomura, T. Watari, and T. Yanagida, Phys. Lett. B 484, 103 (2000), arXiv:hep-ph/0004182

[30] J. E. Kim and H. P. Nilles, Phys. Lett. B 553, 1 (2003), arXiv:hep-ph/0210402.

[31] L. J. Hall, Y. Nomura, and S. J. Oliver, Phys. Rev. Lett. 95, 141302 (2005), arXiv:astro-ph/0503706

[32] J. E. Kim and H. P. Nilles, JCAP 05, 010 (2009), arXiv:0902.3610 [hep-th]

[33] A. Chatzistavrakidis, E. Erfani, H. P. Nilles, and I. Zavala, JCAP 09, 006 (2012) arXiv:1207.1128 [hep-ph]

[34] J. E. Kim, Y. Semertzidis, and S. Tsujikawa, Front. in Phys. 2, 60 (2014) arXiv:1409.2497 [hep-ph]

[35] J. Kang, Y. Gong, G. Cheng, and X. Chen, (2019), 10.1088/1674-4527/20/4/55, arXiv:1912.05926 [astro-ph.CO].

[36] R. Caldwell and E. V. Linder, Phys. Rev. Lett. 95, 141301 (2005), arXiv:astro-ph/0505494.

[37] T. Chiba, A. De Felice, and S. Tsujikawa, Phys. Rev. D 87, 083505 (2013) arXiv:1210.3859 [astro-ph.CO]

[38] S. Tsujikawa, Class. Quant. Grav. 30, 214003 (2013), arXiv:1304.1961 [gr-qc]

[39] J.-B. Durrive, J. Ooba, K. Ichiki, and N. Sugiyama, Phys. Rev. D 97, 043503 (2018), arXiv:1801.09446 [astro-ph.CO]

[40] A. G. Riess, L. Macri, S. Casertano, H. Lampeitl, H. C. Ferguson, A. V. Filippenko, S. W. Jha, W. Li, and R. Chornock, Astrophys. J. 730, 119 (2011), [Erratum: Astrophys.J. 732, 129 (2011)], arXiv:1103.2976 [astro-ph.CO]

[41] A. G. Riess et al., Astrophys. J. 826, 56 (2016), arXiv:1604.01424 [astro-ph.CO].

[42] V. Bonvin et al., Mon. Not. Roy. Astron. Soc. 465, 4914 (2017), arXiv:1607.01790 [astro-ph.CO]

[43] A. G. Riess et al., Astrophys. J. 861, 126 (2018), arXiv:1804.10655 astro-ph.CO]

[44] S. Birrer et al., Mon. Not. Roy. Astron. Soc. 484, 4726 (2019), arXiv:1809.01274 [astro-ph.CO]

[45] A. G. Riess, S. Casertano, W. Yuan, L. M. Macri, and D. Scolnic, Astrophys. J. 876, 85 (2019), arXiv:1903.07603 [astro-ph.CO].

[46] M. Wyman, D. H. Rudd, R. Vanderveld, and W. Hu, Phys. Rev. Lett. 112, 051302 (2014), arXiv:1307.7715 [astro-ph.CO]

[47] E. Di Valentino, A. Melchiorri, and J. Silk, Phys. Lett. B 761, 242 (2016), arXiv:1606.00634 [astro-ph.CO]

[48] G.-B. Zhao et al., Nature Astron. 1, 627 (2017), arXiv:1701.08165 [astro-ph.CO]

[49] E. Di Valentino, A. Melchiorri, E. V. Linder, and J. Silk, Phys. Rev. D 96, 023523 (2017), arXiv:1704.00762 [astro-ph.CO]

[50] E. Di Valentino, A. Melchiorri, and O. Mena, Phys. Rev. D 96, 043503 (2017), arXiv:1704.08342 [astro-ph.CO].

[51] E. Di Valentino, E. V. Linder, and A. Melchiorri, Phys. Rev. D 97, 043528 (2018) arXiv:1710.02153 [astro-ph.CO],

[52] N. Khosravi, S. Baghram, N. Afshordi, and N. Altamirano, Phys. Rev. D 99, 103526 (2019), arXiv:1710.09366 [astroph.CO]

[53] E. Mörtsell and S. Dhawan, JCAP 09, 025 (2018), arXiv:1801.07260 [astro-ph.CO]

[54] V. Poulin, K. K. Boddy, S. Bird, and M. Kamionkowski, Phys. Rev. D 97, 123504 (2018), arXiv:1803.02474 [astro-ph.CO]

[55] K. L. Pandey, T. Karwal, and S. Das, JCAP 07, 026 (2020), arXiv:1902.10636 [astro-ph.CO].

[56] K. Vattis, S. M. Koushiappas, and A. Loeb, Phys. Rev. D 99, 121302 (2019) arXiv:1903.06220 [astro-ph.CO]

[57] S. Alexander and E. McDonough, Phys. Lett. B 797, 134830 (2019), arXiv:1904.08912 [astro-ph.CO].

[58] S. Vagnozzi, Phys. Rev. D 102, 023518 (2020), arXiv:1907.07569 [astro-ph.CO]|

[59] L. Knox and M. Millea, Phys. Rev. D 101, 043533 (2020), arXiv:1908.03663 [astro-ph.CO].

[60] T. Sekiguchi and T. Takahashi, (2020), arXiv:2007.03381 [astro-ph.CO]

[61] T. Karwal and M. Kamionkowski, Phys. Rev. D 94, 103523 (2016) arXiv:1608.01309 [astro-ph.CO].

[62] V. Poulin, T. L. Smith, T. Karwal, and M. Kamionkowski, Phys. Rev. Lett. 122, 221301 (2019), arXiv:1811.04083 [astro-ph.CO].

[63] P. Agrawal, F.-Y. Cyr-Racine, D. Pinner, and L. Randall, (2019), arXiv:1904.01016 [astro-ph.CO].

[64] M.-X. Lin, G. Benevento, W. Hu, and M. Raveri, Phys. Rev. D 100, 063542 (2019), arXiv:1905.12618 [astro-ph.CO].

[65] T. L. Smith, V. Poulin, and M. A. Amin, Phys. Rev. D 101, 063523 (2020) arXiv:1908.06995 [astro-ph.CO]

[66] F. Niedermann and M. S. Sloth, (2019), arXiv:1910.10739 [astro-ph.CO].

[67] K. V. Berghaus and T. Karwal, Phys. Rev. D 101, 083537 (2020), arXiv:1911.06281 [astro-ph.CO]

[68] J. Sakstein and M. Trodden, Phys. Rev. Lett. 124, 161301 (2020), arXiv:1911.11760 [astro-ph.CO].

[69] G. Ye and Y.-S. Piao, Phys. Rev. D 101, 083507 (2020), arXiv:2001.02451 [astro-ph.CO]

[70] F. Niedermann and M. S. Sloth, Phys. Rev. D 102, 063527 (2020), arXiv:2006.06686 [astro-ph.CO]

[71] G. Ye and Y.-S. Piao, Phys. Rev. D 102, 083523 (2020), arXiv:2008.10832 [astro-ph.CO]

[72] T. Fujita, Y. Minami, K. Murai, and H. Nakatsuka, (2020), arXiv:2008.02473 [astro-ph.CO].

[73] L. M. Capparelli, R. R. Caldwell, and A. Melchiorri, Phys. Rev. D 101, 123529 (2020), arXiv:1909.04621 [astro-ph.CO]

[74] D. Harari and P. Sikivie, Phys. Lett. B 289, 67 (1992)

[75] F. Bianchini et al. (SPT), Phys. Rev. D 102, 083504 (2020), arXiv:2006.08061 [astro-ph.CO].

[76] T. Namikawa et al., Phys. Rev. D 101, 083527 (2020), arXiv:2001.10465 [astro-ph.CO].

[77] S. Weinberg, Cosmology (Oxford University Press, 2008).

[78] R. Hlozek, D. Grin, D. J. E. Marsh, and P. G. Ferreira, Phys. Rev. D 91, 103512 (2015), arXiv:1410.2896 [astro-ph.CO]

[79] V. Anastassopoulos et al. (CAST), Nature Phys. 13, 584 (2017), arXiv:1705.02290 [hep-ex]

[80] A. Payez, C. Evoli, T. Fischer, M. Giannotti, A. Mirizzi, and A. Ringwald, JCAP 02, 006 (2015), arXiv:1410.3747 [astro-ph.HE] 
[81] M. Berg, J. P. Conlon, F. Day, N. Jennings, S. Krippendorf, A. J. Powell, and M. Rummel, Astrophys. J. 847, 101 (2017), arXiv:1605.01043 [astro-ph.HE].

[82] R. Bähre et al., JINST 8, T09001 (2013), arXiv:1302.5647 [physics.ins-det]

[83] E. Armengaud et al., JINST 9, T05002 (2014), arXiv:1401.3233 [physics.ins-det]

[84] I. G. Irastorza and J. Redondo, Prog. Part. Nucl. Phys. 102, 89 (2018), arXiv:1801.08127 [hep-ph].

[85] J. P. Conlon, F. Day, N. Jennings, S. Krippendorf, and F. Muia, Mon. Not. Roy. Astron. Soc. 473, 4932 (2018), arXiv:1707.00176 [astro-ph.HE]

[86] V. Poulin, T. L. Smith, D. Grin, T. Karwal, and M. Kamionkowski, Phys. Rev. D 98, 083525 (2018), arXiv:1806.10608 [astro-ph.CO] 